\title{
VII.4 FF4: Relationen alternativer Nachrichtenmedien in der Twittersphäre
}

Nachdem im vorherigen Kapitel eine deskriptive Beschreibung der Netzwerkstrukturen erfolgte, widmet sich das vorliegende Kapitel einem weiteren Analyseschritt der Netzwerkrelationen. Ziel ist es, einen interpretativen Blick auf die Relationen zwischen den Akteur_innen zu werfen, weshalb auf theoretische Annahmen der relationalen Soziologie (vgl. Kapitel IV.1) Bezug genommen wird. Es geht folglich darum, den subjektiven Sinn bzw. Narrative hinter den Beziehungsnetzwerken in Erfahrung zu bringen. Die Relationen zwischen Akteur_innen in der Twittersphäre ergeben sich dabei vor allem mit Hinblick auf Retweets, wie sie netzwerkanalytisch in Kapitel VII.3.2 beschrieben wurden. Basierend auf der Strategie des Theoretical Samplings werden aber auch weitere Tweets der Gesamtdatensätze im Rahmen der qualitativen Inhaltsanalyse ergänzt, die Aufschluss über Bezugnahmen zwischen unterschiedlichen Twitter-Akteur_innen geben. Dies kann in Form von Mentions (@) oder Hashtags (\#) erfolgen, aber auch durch schlichte Nennungen von Akteur_innen innerhalb der Tweets, beispielsweise von Nachrichtenmedien. Als Datenbasis dienen demnach einzelne Tweets, die qualitativ inhaltsanalytisch ausgewertet wurden (Mayring, 1994) (vgl. Kapitel VI.4).

Das Sampling erwies sich als methodische Herausforderung und Balanceakt zwischen theoretischer Sättigung basierend auf einer qualitativ ausgewählten - und entsprechend vergleichsweise kleinen Stichprobe - und dem Umgang mit Datensätzen im sechststelligen Bereich. Unter Verwendung von R wurden die beiden Datensätze deshalb nach folgenden Kriterien vorselektiert. Grundlage für die qualitative Inhaltsanalyse bildeten demnach:

- Die Most-Retweeted-Tweets und Most-Liked-Tweets innerhalb der Datensätze, die Aufschluss über besonders populäre Tweets im Untersuchungszeitraum geben. Diese konnten auf ihre Inhalte, aber auch auf ihre Kommunikationslogiken hin untersucht werden (z. B. Wird auf weitere Akteur_innen verwiesen? Werden Nachrichtenwerte bedient? Zu welchem Zweck werden Retweets eingesetzt?). Fokussiert wurde hierbei vor allem jener Datensatz, der sich auf die URLs alternativer Nachrichtenmedien beschränkt. Im Gesamtdatensatz, der auch professionelle Nachrichtenmedien aus dem DACH-Raum inkludiert, wurden dabei jene Tweets betrachtet, die einen Bezug zu alternativen Nachrichtenmedien aufweisen (z. B. durch Einbettung von URLs). 
- Weiter wurden Tweets, die von alternativen Nachrichtenmedien ${ }^{149}$ abgesendet wurden, zur weiteren Analyse tabellarisch in Excel festgehalten. Da das Ziel und der Zweck qualitativer Analysen nicht darin bestehen, repräsentative Stichproben zu ziehen oder gar Vollerhebungen durchzuführen, wurde basierend auf einer Kontrastierung der Fälle (z. B. besonders populäre Tweets im Gegensatz zu jenen, die kaum Reaktionen erfahren haben) ein Sample gebildet.

- Tweets, in denen die Twitter-Accounts alternativer Nachrichtenmedien markiert wurden, wie auch Tweets alternativer Nachrichtenmedien, die retweetet, also geteilt wurden. Diese Daten sind vor allem deshalb von Interesse, da sie basierend auf den Social-Media-Logiken eine Vernetzung zwischen Akteur_innen deutlich machen.

- Besonders starke Knoten innerhalb der Communities alternativer Nachrichtenmedien wurden für sich detailliert analysiert. Eine Auflistung der stärksten Knotenpunkte je Community findet sich in Anhang 2.

- Besonders berücksichtigt wurden zudem Bezugnahmen zwischen alternativen und professionellen Nachrichtenmedien (Mainstreammedien). Zu diesem Zweck wurden die Datensätze so vorselektiert, dass einerseits alternative Nachrichtenmedien als Sender von Tweets mit Bezugnahme auf professionelle Nachrichtenmedien (in Form von Mentions, namentlicher Nennung innerhalb der Tweets oder Einbettung von URLs) wie auch in umgekehrter Weise (professionelle Nachrichtenmedien mit Bezug auf alternative Nachrichtenmedien) fokussiert werden konnten.

Sämtliche Daten wurden tabellarisch in Excel festgehalten. Kontextinformationen zu den Tweets, wie Urheber_in oder Anzahl an Retweets und Likes, wurden ebenso dokumentiert, um die Beiträge kontextualisieren zu können. Zudem wurden fallweise die eingebetteten URLs gesichtet, um den Inhalt der Tweets einzuordnen. In Excel wurde zudem die Kodierung der Beiträge vorgenommen und in zusätzlichen Spalten festgehalten. Die interpretierten Beiträge basieren auf einer Kontrastierung unter den Beiträgen, mit dem Anspruch, so lange Daten auszuwerten, bis die theoretische Sättigung erzielt ist.

Insgesamt wurden 475 Tweets qualitativ inhaltsanalytisch kodiert. Dies mag in Anbetracht der Datenfülle als kleines Sample erscheinen, muss jedoch aufgrund des qualitativen, interpretativen Ansatzes relativiert werden. In diesem Zusammenhang ist die Analyse von mehreren Hundert Daten sogar relativ umfangreich, konnte aber aufgrund der auf 280 Zeichen beschränkten Tweets realisiert werden. Die Datenerhebung konnte schliesslich unter Realisierung des Anspruchs, eine

149 Hier wurde wiederum auf die Auflistung alternativer Nachrichtenmedien Bezug genommen, die auch für die URL-Suche genutzt wurde (vgl. Anhang 1a). 
theoretische Sättigung zu erreichen, abgeschlossen werden. Die Kodierung (oder: Kategorisierung) der Daten bildet die Basis für die nachfolgende Interpretation. Sämtliche erstellten Kategorien wurden in einem Kategorienschema strukturiert, d. h. die Kategorien wurden gebündelt und in Haupt- und Subkategorien angeordnet. Die Subkategorien dienen dabei zur genaueren Einordnung der Hauptkategorien, da diese in unterschiedlichen Ausprägungen auftreten können. Das Kategorienschema bezieht sich auf sämtliche analysierte Tweets $(n=475)$. Eine Kategorie bezieht sich jeweils auf Regularitäten innerhalb des Datenmaterials. Folgende Tabelle 5 veranschaulicht das entstandene Kategorienschema.

Tabelle 5: Kategorienschema qualitative Inhaltsanalyse «Relationen alternativer Nachrichtenmedien»

\begin{tabular}{|c|c|c|}
\hline Hauptkategorien & Subkategorien & \\
\hline \multirow{7}{*}{$\begin{array}{l}\text { Engagement- } \\
\text { Driver }\end{array}$} & «Klassische» Nachrichtenwerte & Polarisierung \\
\hline & & Skandalisierung \\
\hline & & Personalisierung \\
\hline & & Emotionalisierung \\
\hline & Direkter Bezug auf deutungsmächtige Akteur_innen & Mentions \\
\hline & & Hashtags \\
\hline & & Retweets \\
\hline \multirow{5}{*}{$\begin{array}{l}\text { Demonstration } \\
\text { der Alternative }\end{array}$} & Polarisierung & Thema: Migration \\
\hline & & Thema: Klimawandel \\
\hline & & $\begin{array}{l}\text { Thema: Freiheits- } \\
\text { rechte }\end{array}$ \\
\hline & Diskreditierung der Opposition & \\
\hline & Ironie & \\
\hline \multirow[t]{4}{*}{ Homophilie } & Verlinkung deutungsmächtiger Alternativmedien & \\
\hline & Gemeinschaft durch gemeinsamen «Gegner» & \\
\hline & Gleich und gleich gesellt sich gern: Aufruf zur Vernetzung & \\
\hline & Verteidigung Gleichgesinnter (z. B. Politiker_innen) & \\
\hline $\begin{array}{l}\text { Länderübergrei- } \\
\text { fende Vernetzung }\end{array}$ & Politische Gleichgesinnung als gemeinsamer Nenner & \\
\hline \multirow{2}{*}{$\begin{array}{l}\text { Bezugnahme auf } \\
\text { Politik/Politiker_- } \\
\text { innen }\end{array}$} & Unterstützung: Gleichgesinnte Politiker_innen & \\
\hline & Diffamierung der Opposition & \\
\hline
\end{tabular}




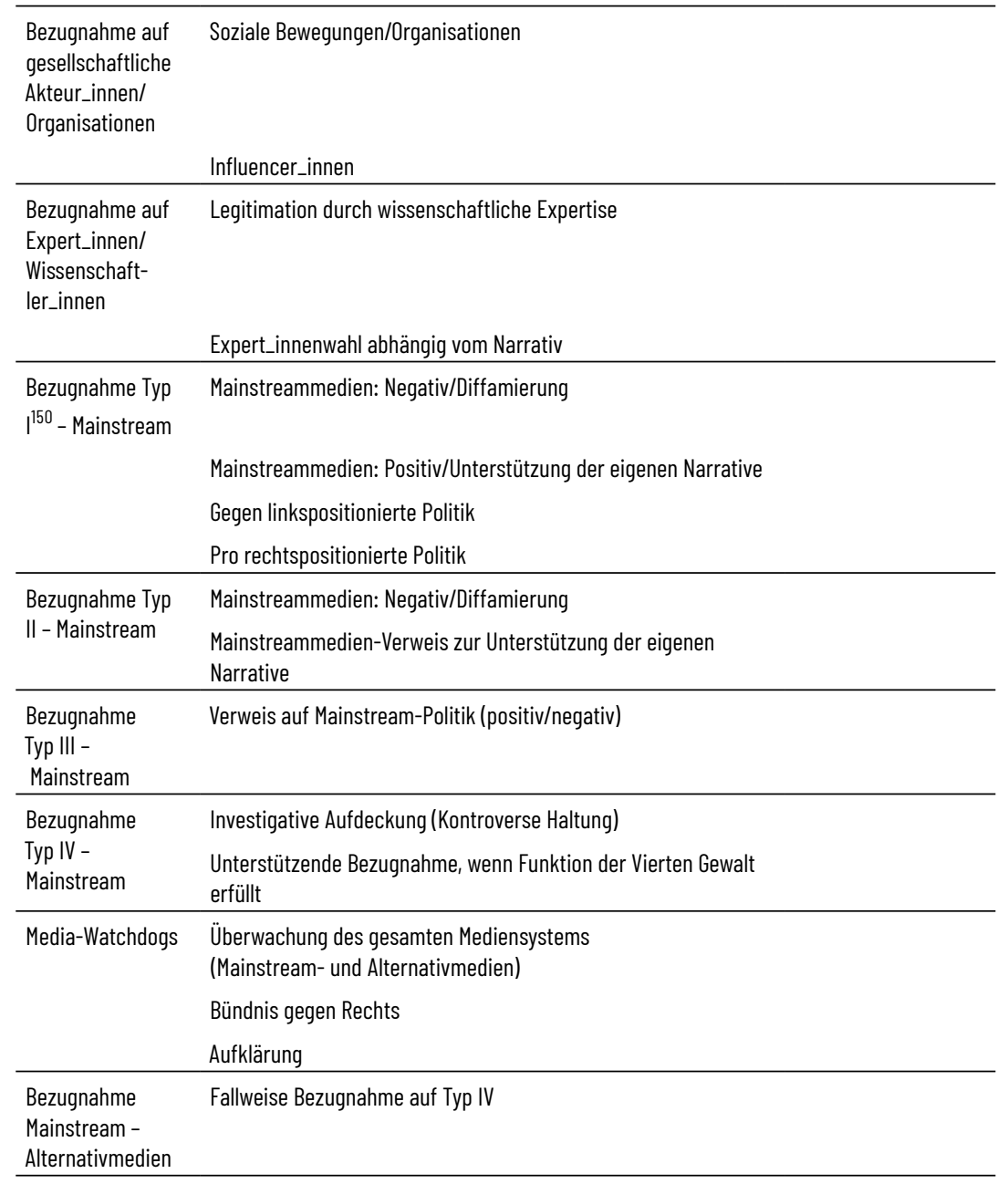

Die kategorienbasierte Interpretation orientiert sich stark an der Typenbildung alternativer Nachrichtenmedien (vgl. Kapitel VII.2). Dies einerseits deshalb, weil - wie bereits innerhalb der strukturellen Netzwerkanalysen ersichtlich wurde die typologische Einordnung alternativer Nachrichtenmedien aus einer netzwerkanalytischen Betrachtung bestätigt werden konnte und weiter vor allem die Relationen zwischen einzelnen Typen und professionellen Nachrichtenmedien von Interesse sind. Andererseits bestätigte sich auch im Rahmen der qualitativen

150 Einordnung in Anlehnung an die Typenbildung alternativer Nachrichtenmedien in Kapitel VII.2. Typ I repräsentiert die «Aufdecker der Mainstreamlügen», Typ II «Verschwörung und Spiritualität», Typ III «Aufstand der Zivilgesellschaft», Typ IV «Die seriöse Alternative». 
Inhaltsanalyse, dass sich die Bezugnahmen zwischen Twitter-Akteur_innen vor allem hinsichtlich der Medientypen unterscheiden. Die in Kapitel VII.2 beschriebenen inhaltlichen Fokusse der vier Typen stimmen zudem auch mit den Themen überein, die innerhalb der Twittersphäre von den alternativen Nachrichtenmedien fokussiert wurden, was für eine Validität der Analyse der Websites dieser Nachrichtenmedien, unter Verwendung des methodischen Ansatzes der Grounded Theory, spricht. Insofern erwies sich bei Typ I eine Auflehnung gegen die Mainstreammedien und linksorientierte Politik als prägend, was sich vor allem im Kontext des Klimadiskurses verdeutlichte. Typ II und Typ III sind zwar aufgrund ihrer eher laienhaften Aufmachung im Bereich des Pseudo-Journalismus unterrepräsentiert, jedoch liessen sich auch hier eine verschwörungstheoretische Tendenz von Typ II und ein Protestcharakter gegen die herrschenden Eliten bei Typ III feststellen. Prägend für Typ IV war auch in dem von Twitter gesampelten Datenmaterial die investigativ-journalistische Ausrichtung im Sinne von Watchdogs des gesamten Mediensystems.

Die folgenden Subkapitel dienen der Interpretation der einzelnen Hauptkategorien, die mit beispielhaften Zitaten der Tweets belegt werden. In Kapitel VII.4.14 werden die zentralen Erkenntnisse schliesslich resümiert.

\section{VII.4.1 Engagement-Driver}

Bevor auf die konkreten Bezugnahmen zwischen unterschiedlichen Twitter-Akteur_innen eingegangen wird, nimmt diese Kategorie Bezug auf Twitter-Medienlogiken oder auch Social Media Logics respektive Plattformlogiken (Eisenegger, 2021; Klinger \& Svensson, 2015; van Dijck \& Poell, 2013). In diesem Zusammenhang stellt sich erstens die Frage, von welchen Logiken Beiträge auf Twitter geprägt sind, die hohe Resonanz - in Form von Favorisierungen oder Retweets - auslösen. Auch wenn davon auszugehen ist, dass Social-Media-Plattformen ähnlichen Logiken folgen, widmet sich diese Kategorie ausschliesslich dem empirischen Ausschnitt Twitter und der Forschungsfrage entsprechend Beiträgen, die von alternativen Nachrichtenmedien versendet werden oder zumindest einen Bezug zu alternativen Nachrichtenmedien aufweisen. Eine Analyse der resonanzreichsten Tweets - unter kontrastierender Betrachtung resonanzschwacher Beiträge - innerhalb der zugrundeliegenden Datensätze zeigte zunächst deutlich, dass klassische Nachrichtenwerte bedient werden, die auch im traditionellen Journalismus eine wesentliche Rolle einnehmen. In Anbetracht des Untersuchungsgegenstandes nicht verwunderlich, ist es vor allem der Nachrichtenwert der Polarisierung, der sich im Material klar herauskristallisierte. Wie bereits veranschaulicht, erwiesen sich im Datenmaterial vor allem alternative Nachrichtenmedien des Typs I und Typs IV als besonders präsent. Dies sind jene beiden Typen, die sich am stärksten voneinander unterscheiden. Während Typ I eine - aus normativer Sicht 
- Medien- und Politikkritik mit rechtspopulistischen Tendenzen äussert und sich das Ziel setzt, «Lügen des Mainstreams» aufzudecken, agiert Typ IV aus einem demokratiebasierten, eher linksorientierten Standpunkt, Investigativ-Journalismus zu betreiben und somit der mangelnden Rechercheleistung im kommerzialisierten Mainstream-Mediensystem nachzukommen.

Polarisierungstendenzen werden daher vor allem auf politisch-bewertender Ebene ausgehend von den alternativen Nachrichtenmedien ersichtlich - und dies sowohl im linken wie auch im rechten Lager. So lautet beispielsweise ein besonders populärer Tweet mit fast 1'000 Retweets und doppelt so vielen Favorisierungen ${ }^{151}$ : «RT@Volksverpetzer: Die Seite 〈Wir werden sie jagen〉 zeigt erschreckend alle rassistischen und menschenverachtenden Zitate der\#AfD - Sie will so tun als sei sie bürgerlich. Erinnert alle daran, dass das nicht so ist.https://t.co/qQMgDBDWwi@Gegen_die_AfD @hogesatzbau» ${ }^{152}$. Der Tweet stammt von dem Twitter-Account des alternativen Nachrichtenmediums Volksverpetzer, das - wie bereits an einer anderen Stelle erläutert - als Media-Watchdog im Sinne eines Metadiskurses über Medien fungiert und am ehesten dem Typ IV alternativer Nachrichtenmedien zuzuordnen ist. Durch die Verwendung von Begrifflichkeiten wie «rassistisch» oder «Menschenverachtend» wird eine deutlich polarisierte bzw. oppositionelle Haltung zu rechter Politik deutlich, in diesem Fall mit Bezug auf die AfD. Die Phrase «Erinnert alle daran, dass das nicht so ist» offenbart zudem einerseits einen Gemeinschaftscharakter unter Gleichgesinnten, verbunden mit einer Aufklärungs- und Mobilisierungsfunktion. Verdeutlicht wird dies durch Verlinkungen in Form von Mentions (@) der Twitter-Accounts Gegen die AfD und - einem ähnlichen Narrativ folgend - hogesatzbau, der sich selbst in ironischer Art als «Initiative gegen Rechts-Schreibung» beschreibt. In ähnlicher Weise, aber aus dem rechten politischen Lager stammend, erzeugt ein Tweet von Roland Tichy (Herausgeber von Tichys Einblick) Resonanz und wird beispielsweise gleichzeitig vom Alternativmedium Sezession retweetet: «RT@RolandTichy: 〈Antifaschismus〉-wie eine verfassungsfeindliche Organisation und Denkweise in den Bundestag eindringt, Geschichte verfälscht und manipuliert. Wehren wir uns endlich. https://t.co/sQroCWKIhI» ${ }^{153}$. Dieses beispielhafte Zitat verdeutlicht einerseits den Anspruch von alternativen Nachrichtenmedien des Typs I, Lügen und Manipulationen aufzudecken. Andererseits wird eine klare oppositionelle Haltung gegenüber politischer Linksorientierung deutlich. Aufschluss über den Inhalt des Tweets gibt der beigefügte URL, der auf einen Artikel in Tichys Einblick verweist, der sich mit der antifaschistischen Bewegung (Antifa) auseinan-

151 Bei den folgenden Zitaten handelt es sich um beispielhafte Quotes aus dem analysierten Datenmaterial. Sie wurden mit dem Anspruch ausgewählt, die jeweiligen Kategorien zu repräsentieren, und verdeutlichen Regularitäten innerhalb des Datenkorpus.

152 https://twitter.com/i/web/status/1185837956961067008 (Volksverpetzer, 21.10.2019)

153 https://twitter.com/i/web/status/1179681957485203456 (Sezession im Netz, 03.10.2019) 
dersetzt und vor Linksextremismus warnt. Wie auch im Zitat von Volksverpetzer lässt sich innerhalb des Tweets auch eine gemeinschaftsbildende Komponente («Wehren wir uns endlich») erkennen. Beide hier zitierten Tweets erwecken den Eindruck eines dichotomen Verhältnisses zwischen Links und Rechts, wobei starke, aufmerksamkeitsgenerierende Worte wie «menschenverachtend» oder «manipuliert» verwendet werden, um die oppositionelle Haltung deutlich zu machen - im Sinne von: Wir gegen die Anderen.

Auch der klassische Nachrichtenwert der Skandalisierung ist einerseits Teil der Kommunikationslogiken von alternativen Nachrichtenmedien und erzeugt andererseits Aufmerksamkeit bzw. Engagement. So erzielt beispielsweise der Twitter-Account wach_jetzt_auf vor allem Resonanz durch die Verlinkung von URLs alternativer Nachrichtenmedien des Typs I und wählt hierfür skandalisierende Titel wie beispielsweise: «! Finnische Migrationsbehörde: Großteil der Schutzsuchenden aus dem Irak sind Fahnenflüchtige ! https://t.co/oHvzEbN3zr via @jouwatch» ${ }^{154}$, oder: «! Betrug und Terror durch gefälschte Identitäten: Wer bin ich - und wenn ja, wie viele? ! $\quad$ https://t.co/KLArACLHwq \#anonymousnews via @anoynews» ${ }^{155}$. Beide Tweets, die sich auf Beiträge von Journalistenwatch und Anonymousnews beziehen, setzen sich mit der Flüchtlingsthematik auseinander. Dabei werden Flüchtlinge diskreditiert bzw. deren Motive skandalisiert, indem sie als «Fahnenflüchtlinge» bzw. Deserteure bezeichnet werden, deren Identitäten verfälscht seien und mit Terror in Verbindung stünden. In beiden Tweets wird durch den Einsatz von Emoticons zusätzlich Aufmerksamkeit erregt, wonach die Visualisierung eines Warndreieckes auf Gefahr hinweiset. Im Zuge der Verbreitung von skandalisierenden Beiträgen, die massgeblich von Typ I eingesetzt werden, findet zudem häufig ein Bezug zum professionellen Journalismus statt. Dies ist insbesondere dann der Fall, wenn das eigene Narrativ durch die Berichterstattung der Mainstreammedien gestützt wird. Häufig findet somit ein Bezug zu Boulevardmedien statt, die schliesslich selbst Nachrichtenwerte wie Skandalisierung bedienen. Auch hier wird häufig auf den Flüchtlingsdiskurs Bezug genommen, wie sich anhand des folgenden Beitrags zeigt: «Junger Syrer sticht 38-jährigem Österreicher Messer in den Hals https://t.co/ J9OHCW8O2I» ${ }^{156}$. Der Tweet wurde von dem Account staatsversagen publiziert, der vom alternativen Nachrichtenmedium Poltikversagen.net $t^{157}$ bewirtschaftet wird, und verweist auf die Online-Ausgabe des österreichischen Boulevardmediums Kronen Zeitung. Während der Artikel selbst in der Überschrift nicht auf die Nationali-

\footnotetext{
154 https://twitter.com/jouwatch/status/1182944039873798144 (Journalistenwatch, 12.10.2019)

155 https://www.anonymousnews.ru/2019/10/11/gefaelschte-identitaeten-wer-bin-ich-und-wennja-wie-viele/ (Anonymousnews, 12.10.2019)

156 https://t.co/]9OHCW8O2l (Staatsversagen, 16.09.2019)

157 http://www.politikversagen.net (Stand: 07.10.2020)
} 
täten von Täter und Opfer hinweist, erregt der Tweet gerade dadurch Aufmerksamkeit und verweist gleichzeitig auf potentielle Gewaltprobleme durch Migrant_innen.

Weiter dient der Nachrichtenwert der Personalisierung als Engagement-Driver. Diese Personalisierung kann in unterschiedlicher Form auftreten. So ist es beispielsweise der Struktur von Social Media, im Sinne einer Vernetzung von Einzelpersonen auch im (alternativ-)journalistischen Bereich, geschuldet, dass häufig auf die Journalist_innen selbst Bezug genommen wird, die einen Artikel verfasst haben. Diese wiederum verlinken ihre eigenen Beiträge unter Bezugnahme auf das Nachrichtenmedium, in dem der Artikel erschienen ist. Dies zeigte sich unabhängig vom (Alternativ-)Medientyp. Beispielhaft sei eine Verlinkung des Republik-Journalisten Daniel Binswanger vom Magazin selbst genannt: «RT@DBinswanger: Wie können wir die wachsende Ungleich heit in den Griffbekommen? Warum ist die heutige Linke in der Defensive? Was ist progressive Politik? Der Star-Ökonom Thomas Piketty hat ein monumentales neues Werk veröffentlicht. Und gibt Antworten. @RepublikMagazin» ${ }^{158}$. Personalisierung als Nachrichtenwert wird aber vor allem eingesetzt, um Organisationen, wie beispielsweise politische Parteien oder auch Non-Profit-Organisationen, ein Gesicht zu geben. Neben der namentlichen Nennung von politischen Akteur innen (so beispielsweise ein Tweet von NewsAustria, dem Account der Website Oesterreich.press ${ }^{159}$ : « \#Kann Kurz mit den Grünen? - FAZ - Frankfurter Allgemeine Zeitung... https://t.co/W8kjAZK5VI» ${ }^{160}$, bei dem klar auf den österreichischen Bundeskanzler Sebastian Kurz eingegangen und die Grüne Partei ohne persönlichen Bezug erwähnt wird), sticht im analysierten Datenmaterial vor allem die Konnotation von Greta Thunberg mit der Fridays-for-Future-Bewegung - sowohl in positiv bestätigendem als auch negativ abwertendem Sinn - heraus: «RT @Volksverpetzer: Die \#BILD lässt einen Politologen mit leicht widerlegbaren Argumenten Stimmung gegen \#GretaThunberg und @FridayForFuture machen und nennt ihn «Forscher〉, um es seriös erscheinen zu lassen. [...]», oder: «Klima-Göre Thunberg droht: 〈Wir werden euch nicht davonkommen lassen!> https://t.co/Lfm6qo8Y9b \#anonymousnews via @anoynews» (retweetet von deutsch365). ${ }^{161}$ Thunberg gilt dabei als die Person, die die junge Klimabewegung repräsentiert - dabei kann sie in der Öffentlichkeit selbst als polarisierende Person betrachtet werden, wodurch die Diskrepanz zwischen unterschiedlichen alternativen Nachrichtenmedien (oder auch alternativen und professionellen Nachrichtenmedien) verdeutlicht wird.

158 https://twitter.com/i/web/status/1182950693155540992 (Republik Magazin, 12.10.2019)

159 Mittlerweile (Stand: 07.10.2020) abrufbar unter https://querdenken.international (Alternatives Nachrichtenmedium des Typs I)

160 https://oesterreich.press/?p=57245 (Oesterreich.press, 06.10.2019)

161 https://twitter.com/i/web/status/1178607984110444545 (Volksverpetzer, 30.09.2019); https://www. anonymousnews.ru/2019/09/23/greta-thunberg-wirre-drohungen-un-klimagipfel-new-york/ (AnonymousNews, 23.09.2019) 
Als letzter klassischer Nachrichtenwert, der im Rahmen dieser Kategorie beschrieben wird, ist die Emotionalisierung zu nennen. Social Media werden in der aktuellen Forschung auch als Emotionsmedien beschrieben, wonach sich vor allem affektive Beiträge auf Digitalplattformen verbreiten (Schwaiger, 2019). Dieser Befund konnte auch im Datenmaterial der vorliegenden Studie bestätigt werden. Entsprechend erzielen emotionale Beiträge Aufmerksamkeit, beispielsweise in Form von Geschichten aus der Zivilgesellschaft, die medial veröffentlicht werden. Beispielhaft sei ein Tweet des österreichischen Mediums Wochenblick (Typ I) aufgeführt:

RT @wochenblickat: Weil er ein Kreuz um dem Hals trug, durfte ein Deutscher nicht in einer \#Essener Disco feiern. «lch bin Christ. Wenn ich in meinem eigenen Land kein Kreuz mehr tragen darf, dann läuft etwas nicht richtig», so der Mann, der bisher Stammgast in dem Club war. ${ }^{162}$

Eine weitere Möglichkeit der Emotionalisierung ist der Einsatz von Emoticons, die Gefühle bzw. Emotionen hinter Textbeiträgen verstärken sollen: «RT@ wach_jetzt_auf: ! jetzt geht's richtig los ! Öffentlich Rechtliches Fernsehen wirbt für Konvertierung zum Islam. W. Warum müssen wir das noch finanzieren? Schluss damit 2 ! $»^{163}$. Beide Zitate beziehen sich - wie häufig bei Alternativmedientyp I - auf die Migrationsthematik. Das zweite Zitat thematisiert zudem die Kritik am öffentlich-rechtlichen Rundfunk. Eine Emotionalisierung findet daher vor allem in Bezug auf den Ausdruck von Ärger oder Besorgnis statt, gleichzeitig verbunden mit einer Skandalisierung verschiedener Akteur_innen, seien es Migrant_innen oder das Mediensystem selbst.

Abgesehen von klassischen Nachrichtenwerten oder Medienlogiken, die im Datenmaterial identifiziert wurden, konnten auch Indizien für den Einsatz von Social-Media- respektive Plattformlogiken herausdestilliert werden. Dabei beziehe ich mich auf die Twitter-spezifischen Kommunikationsmöglichkeiten, die eine Vernetzung von Akteur_innen (Vernetzung im Sinne einer Social-Media-Logik für sich) ermöglichen. Darunter ist erstens der Einsatz von Retweets zu nennen, zweitens die Markierung von Twitter-Accounts über Mentions und drittens der Einsatz von Hashtags, um bestimmte Themen oder Wörter an sich zu markieren. Im Zuge der Analyse wurde offensichtlich, dass die drei Funktionen in unterschiedlicher Weise und (womöglich) mit unterschiedlichem Ziel eingesetzt werden. Retweets bzw. das Teilen von Beiträgen sind bzw. ist in diesem Zusammenhang der grösste Popularitätstreiber von Beiträgen, da die Reichweite von Einzelbeiträgen dadurch noch über weitere Follower_innenkreise von den retweetenden Akteur_innen erhöht wird. Dabei werden vor allem Beiträ-

162 https://twitter.com/i/web/status/1183723406069399553 (Wochenblick, 15.10.2019) 163 https://twitter.com/i/web/status/1173248303527616519 (wach_jetzt_auf, 16.09.2019) 
ge retweetet, die - wie erläutert - Nachrichtenwerte bedienen. Mentions und Hashtags werden wiederum in unterschiedlicher Weise respektive mit unterschiedlicher Logik eingesetzt. Mentions dienen der direkten Bezugnahme auf andere Twitter-Akteur_innen. Dem Untersuchungsphänomen entsprechend sind in diesem Fall häufig Mentions von Journalist_innen vorzufinden, die die Beiträge verfasst haben und auf die durch Einbettung von URLs verwiesen wird. Mentions können zudem verwendet werden, um den Diskurs anzufeuern, beispielsweise, indem Politiker_innen, Expert_innen oder weitere Personen des öffentlichen Lebens direkt angesprochen werden - in positiver wie auch negativer Weise: «RT @Die_Insider: Mh. Also, was soll man zur Rolleder@AfDimBundestag und ihrer Mitarbeiter noch alles sagen, bevor es in allen Köpfen ankommt?! @ StBrandnerretweetetsowas.\#halleo910@politicalbeauty@Volksverpetzerhttps://t.co/ lqnqmnıbRz» ${ }^{164}$. Bei diesem Zitat handelt es sich um einen Tweet der als selbst bezeichneten «Recherchegruppe über die AfD» Die Insider, die auf unterschiedliche Twitter-Akteur_innen Bezug nimmt: u. a. auf den offiziellen Account der AfD-Fraktion im deutschen Bundestag und den Rechtsanwalt Stephan Brandner, der einen Beitrag des alternativen Mediums Hartes Geld teilte, in dem auf die deutsche Herkunft der Opfer des Halle-Amoklaufs in einer jüdischen Synagoge 2019 verwiesen wird. Ebenso werden der Account des WatchdogMediums Volksverpetzer und die Organisation Political Beauty verlinkt, die sich für politischen Widerstand einsetzt. Mentions dienen dementsprechend auch dazu, potentiell interessierte Akteur_innen über einen Beitrag zu informieren, im Sinne eines Vernetzungsgedankens Gleichgesinnter. Ebenso verdeutlicht dieses beispielhafte Zitat, dass Hashtags vor allem zur Einordnung von Ereignissen verwendet werden, wie in diesem Fall das Ereignis von Halle 2019. Hashtags werden indes nicht dazu benutzt, um in den Diskurs mit Akteur_innen zu treten, sondern um eine Einordnung bestimmter Themen vorzunehmen. Dies kann als medial angeeignetes Wissen interpretiert werden, wonach die Twitter-User_innen die Funktionen bewusst einsetzen. Ein Hashtag dient dabei vorwiegend der gesammelten Suche von Beiträgen zu einem bestimmten Thema respektive Hashtag. Das Engagement bezüglich bestimmter Twitter-Posts wird folglich insbesondere durch Retweets und Mentions vorangetrieben. In beiden Fällen handelt es sich aber nicht unbedingt um einen positiven, unterstützenden Ausdruck, sondern durchaus auch um ein Mittel der Provokation, um einen Diskurs anzuzetteln, oder aber eine Art Aufklärungsfunktion. Ein Beispiel für eine negative bzw. nichtunterstützende Bezugnahme ist jener Retweet des Accounts ddbnews (Typ I/II), mit Verlinkung eines Artikels von oe24.at, in dem über die «Grüne Korruptionsaffäre» berichtet wird, wobei der Account der Grünen direkt markiert wird: «@Gruene_Austria Ich lese bei den Grünen nichts 
über derartige Beiträge, die fast täglich erscheinen. https://t.co/heK61bzlrK» ${ }^{165}$. Eine Aufklärungsfunktion und gleichzeitig negative Bezugnahme tritt beispielhaft in folgendem Beitrag zu Tage, der vom alternativen Nachrichtenmedium Kontrast.at (Typ III) veröffentlicht wurde: «@sebastiankurz meint es gibt nichts mehr zum Aufklären beim \#BVT. Hier zur Auffrischung nur einer von vielen Skandalen um den BVT. \#wahli9. https://t.co/oGZW4yfisQ» ${ }^{166}$. An diesem Punkt kann bereits festgehalten werden, dass Retweets und Mentions in unterschiedlicher Weise auftreten können und gleichbedeutend nicht für eine unterstützende Haltung gegenüber den verlinkten Akteur_innen stehen müssen. Dies ist vor allem deshalb von Bedeutung, da rein strukturelle Betrachtungen von Netzwerken keine derartige Unterscheidung vornehmen und allenfalls $\mathrm{zu}$ voreiligen Schlüssen führen können.

\section{VII.4.2 Demonstration der Alternative}

Die Demonstration der Alternative kann als wesentliches Kriterium genannt werden, wenn der Begriff der alternativen Nachrichtenmedien definiert werden soll. Schliesslich deutet der Begriff selbst auf ein relationales Verhältnis hin - in diesem Fall zwischen Gegenöffentlichkeit (Alternative) und Öffentlichkeit (Mainstream). Diese Demonstration kann in unterschiedlicher Weise in Erscheinung treten und wurde im Zuge der Analyse mit drei Subkategorien zusammengefasst. Die erste Sub-Kategorie in ist jene der Polarisierung, die bereits im Rahmen der Kategorie «Engagement-Driver» (vgl. Kapitel VII.4.1) als Nachrichtenwert thematisiert wurde. Dennoch soll sie an dieser Stelle aus einer weiteren Perspektive besprochen werden. Die Polarisierung bezüglich der «Demonstration der Alternative» verdeutlicht sich dabei vor allem im Zusammenhang der von den Medien besprochenen Themen. Am präsentesten im analysierten Material waren die Themen Migration und Klimawandel, entsprechend in einem politischen Kontext, zumal der Erhebungszeitraum mit politischen Wahlen in den drei Untersuchungsländern zusammenfiel. Eine Polarisierung findet dahingehend insbesondere in einer dichotomen Sichtweise statt, indem entweder linke respektive rechte Politik abgewertet wird. Dies erfolgt beispielsweise im Kontext der Migrationsthematik:

RT @SiNetz: Das sind jene Leute, die bei islamistischen linksextremen Tätern, die unzählige Anschläge in Europa und der Welt begehen, beschwören, daß das alles mit nichts zu tun habe. Nun, bei einem US-Internet-radikalisierten \&

165 https://t.co/heK61bzIrK (OE24.at, 21.09.2019)

166 https://twitter.com/i/web/status/1174399722506936321 (Kontrast.at, 18.09.2019) 
wahnsinnigen Einzeltäter, werden Verbindungen gezogen. \#Halle https://t.co/ FNarCdo5qb ${ }^{167}$

Der Beitrag stammt vom Medium Sezession und wurde vom Twitter-Accout einprozent verlinkt (beide Typ I). Auch dieser Post bezieht sich auf das Halle-Attentat, beigefügt ist ein Screenshot eines Artikels von Die Zeit mit dem Titel «Anschlag in Halle: Er ist nicht allein», in dem auf das generelle Problem des Antisemitismus vor allem in AfD-Kreisen hingewiesen wird ${ }^{168}$. Innerhalb des Tweets wird somit eine Abgrenzung zum medialen Mainstream vorgenommen, gleichzeitig wird aber auch eine oppositionelle Haltung gegenüber Links deutlich, die durch den Begriff «linksextreme» rhetorisch verstärkt wird. Die Verwendung des Begriffes «linksextreme» deutet darüber hinaus auf dessen Verwendung als eine Art Kampfbegriff hin. Dies zeigt sich auch dann, wenn vom gleichen Typ alternativer Nachrichtenmedien (Typ I) der Begriff «rechtsextrem» in ironischer Weise unter Anführungszeichen gesetzt wird: «RT @Junge_Freiheit: Das Verwaltungsgericht Köln hat dem Bundesamt für Verfassungsschutz untersagt, die Identitäre Bewegung als «gesichert rechtsextremistische Bestrebung zu bezeichnen. Das Gericht gab dem Antrag auf Erlaß einer einstweiligen Anordnung statt.» ${ }^{169}$ Aber auch in oppositioneller Weise findet eine Abgrenzung von rechter Politik bzw. Rechtsextremismus statt, wie anhand dieses Tweets beispielhaft veranschaulicht wird: «RT @Volksverpetzer: Dieser Artikel ist der Beweis, dass \#AfD-Rechtsextremismus inzwischen beim Springer-Verlag vollkommen salonfähig geworden ist. Wir zerlegen alle Lügen und wirren Unterstellungen von \#Springer-Chef \#Döpfner. \#Halle https://t.co/jYkv6gLlRE \#Halle0910@Gegen_die_AfD» ${ }^{170}$. In ähnlicher Weise findet sich dieses Narrativ in der Klimathematik, indem stark polarisierte Meinungen und keine zwiespältigen Haltungen aufeinandertreffen. Als Beispiel hierfür sei ein Beitrag von wach_jetzt_ auf mit Verlinkung auf einen Artikel der Jungen Freiheit (beide Typ I) aufgeführt: "! Klimajugend-Gruppenzwang an deutschen Schulen: 〈Wie in der DDR〉 https:// ¿๐ t.co/JFGcc827MF via @jouwatch» ${ }^{171}$. Obwohl das analysierte Material klar von diesen beiden Themen dominiert ist, erwies sich auch das Thema «Freiheitsrechte» als prägend für alternative Nachrichtenmedien, wodurch eine polarisierte Haltung gegenüber dem (politischen und medialen) Mainstream deutlich gemacht wird:

\footnotetext{
167 https://twitter.com/i/web/status/1182208582516916224 (Sezession im Netz, 10.10.2019)

168 https://www.zeit.de/politik/deutschland/2019-10/anschlag-halle-synagoge-antisemitsmusrechtspopulismus/seite-2 (Stand: 10.10.2020)

169 https://twitter.com/i/web/status/1176884778097872896 (Junge Freiheit, 25.09.2019)

170 https://twitter.com/i/web/status/1182706604518629377 (Volksverpetzer, 11.10.2019)

171 https://t.co/JFGcc827MF (Journalistenwatch, 19.09.2019)
} 
@anc_de@tagesschau @heutejournal @SZ @SPIECEL_Politik @sternde@ FAZ_Politik@derfreitag@ndaktuell @taz_news das sind alles keine deutsche Medien. Wenn ihr DEUTSCHE Medien schon dann meint, müsst ihr zum einzigen deutschen Medium auch kommen! Und das sind nun mal wir. Das ddbradio und ddbnews. Alles andere sind Medien der BRD und NWO und die wollen keine Freiheit ! https://t.co/1ZpOPmiFWk ${ }^{172}$

In diesem Beitrag werden sämtliche deutsche Medientitel des professionellen Journalismus vom Account ddbnews (Typ I/II) verlinkt, allerdings mit einer negativen Konnotation. Das Alternativmedium positioniert sich dabei als einzig wahres deutsches Medium, das für Freiheitsrechte einstehe. Ein verschwörungstheoretischer Charakter des Mediums zeigt sich durch die Begriffsverwendung «BRD» und «NWO». «NWO» bezieht sich auf die Verschwörung der «New World Order», die von einer elitengetriebenen, geplanten Weltregierung ausgeht. In diesem Kontext wird postuliert, dass die Bundesrepublik Deutschland (BRD) kein souveräner Staat sei ${ }^{173}$. Aber auch in anderen Zusammenhängen (und nicht ausschliesslich bezüglich Pressefreiheit) appellieren alternative Nachrichtenmedien an Freiheitsrechte, so beispielsweise auch in Bezug auf Vorratsdatenspeicherung, mit einer klaren oppositionellen Haltung zur «Mainstream»-Politik: «RT @chaosupdates: Innenminister \#Seehofer bringt die \#Vorratsdatenspeicherung wieder ins Gespräch: Jeder soll aufgezeichnet werden. Das ist eine politische Bankrotterklärung. https://t.co/nmiDm8TLBG» ${ }^{174}$.

Als weitere Kategorie im Zusammenhang mit der «Demonstration der Alternative» resultierte die «Diskreditierung der Opposition». Diese Kategorie ist davon geprägt, dass ein sachlicher, argumentbasierter Diskurs zwischen alternativen Nachrichtenmedien und der hegemonialen Öffentlichkeit häufig nicht gegeben ist, vorwiegend ausgehend von Typ I, was sich bereits in der typologischen Untersuchung feststellen liess (vgl. Kapitel VII.2). Dies kann beispielsweise durch Beleidigungen geschehen, wie anhand dieses Tweets von Journalistenwatch ersichtlich: «5 grüne Umweltsünden - und noch viel viel mehr grüner Hirnquark https://t. co/W4M6Z7C9Ks» ${ }^{175}$. Dabei geht es vor allem um eine Abwertung der Opposition. Auch die Sub-Kategorie «Ironie» steht mit diesen Diskreditierungsbemühungen in Zusammenhang. Ironische Tweets werden so vorwiegend dafür eingesetzt, um sich über die Opposition lächerlich zu machen. Beispielhaft zeigt dies ein Ret-

172 https://twitter.com/i/web/status/1185117183157903361 (ddbnews, 18.10.2019)

173 Vgl. z. B. https://www.br.de/puls/themen/welt/ist-deutschland-ein-souveraener-staat-100. html (Stand: 10.10.2020)

174 https://twitter.com/i/web/status/1184119509860401152 (Cicero, 15.10.2019)

175 https://www.journalistenwatch.com/2019/09/20/5-gruene-umweltsuenden-und-noch-vielviel-mehr-gruener-hirnquark/ (Journalistenwatch, 20.09.2019) 
weet der Politikstube, der sich auf einen Beitrag der AfD-Politikerin Alice Weidel bezieht: «RT @Alice_Weidel: Medien:〈Deutscher hat seine Frau <angefahren〉 und sie anschließend mit einer Axt erschlagen. Wie bitte? Es war ein eingebürgerter Tunesier, der die Frau - die ihn bereits mehrfach anzeigte - praktisch enthauptete! \#Limburg \#Migration \#Staatsversagen https://t.co/OrvnGsnpxk» ${ }^{176}$. Gerade der ironische Einsatz von Anführungszeichen ist für alternative Nachrichtenmedien des Typs I wie auch gleichgesinnte Akteur_innen prägend. Aber auch abgesehen von Typ I werden ironische Beiträge genutzt, um die oppositionelle Haltung deutlich zu machen, so beispielsweise vom Watchdog-Medium Volksverpetzer (Typ IV): «RT @Volksverpetzer: Heute ist \#Klimastreik! Das heißt, auch viele Klimawandelleugner werden mit den immer gleichen, falschen Argumenten unterwegs sein. Damit ihr vorbereitet seid, haben wir dieses Bullshit-Bingo mitsamt Quellen vorbereitet. https://t.co/vKaRtEKZtg \#Alle FuersKlima \#FridaysForFuture» ${ }^{177}$. Das «Bullshit-Bingo» ${ }^{178}$ sammelt dabei Aussagen von Klimawandelleugner_innen (z. B. «Es ist gar nicht wärmer geworden»), die für jene Gruppe typisch sind.

Die «Demonstration der Alternative» basiert dementsprechend weniger auf einem argumentbasierten Diskurs und wird vielmehr durch stark polarisierende Haltungen über unterschiedliche (aber wenige und vor allem politisch resonanzreiche) Themen hinweg in einem häufig diskreditierenden, ironischen Diskursstil verdeutlicht. An dieser Stelle muss jedoch berücksichtigt werden, dass diese Kategorie vorwiegend in den Tweets von Typ I bzw. II vorgefunden wurde. Typ III, der kaum auf Resonanz in der Twittersphäre stösst bzw. generell (basierend auf dem zugrundeliegenden Sample dieser Studie) auf Twitter selten vertreten ist, präsentiert seine alternative Haltung vielmehr basierend auf mobilisierenden Beiträgen mit Protest-Charakter, wie an diesem Retweet durch die Junge Welt ersichtlich: «RT @iL_Hamburg: «Es muss sofort gehandelt werden, um die \#Klimakatastrophe noch aufzuhalten. Deshalb wollen wir jetzt den Druck auf Politik und Konzerne erhöhen. Demonstrieren und Streiken allein reicht uns nicht mehr.> @Sitzenbleibenhh in @jungewelt \#Klimastreik https://t.co/LgToakt60i» ${ }^{179}$. Oder aber, indem (rechts-)konservative Politik abgelehnt wird: «\#Faktencheck: \#Kurz sagt, dem Fachkräftemangel muss man durch Ausbildung und nicht durch Zuzug begegnen. Real hat \#Schwarzblau die AMS-Budgets gekürzt und die \#Zuwanderung in den Arbeitsmarkt ist hoch wie nie. \#orfwahl19 \#NRW2019 https://t.co/NxiGQ95uxX» ${ }^{180}$. Typ IV («Die seriöse Alternative») ist weitestgehend von einem sachlichen Diskurs geprägt; eine «Demonstration der Alternative» konnte zumindest im Twitter-Diskurs nicht festgestellt werden, mit

176 https://twitter.com/i/web/status/1188009950842408961 (Weidel, 26.10.2019)

177 https://twitter.com/i/web/status/1174989474922684416 (Volksverpetzer, 20.09.2019)

178 https://www.volksverpetzer.de/klima/klimawandelleugnern-bsbingo/ (Stand: 10.10.2020)

179 https://twitter.com/i/web/status/1174232276378509313 (iL_Hamburg, 18.09.2019)

180 https://twitter.com/i/web/status/1174402671794708480 (Kontrast.at, 18.09.2019) 
Ausnahme der als «Watchdogs» bezeichneten Websites wie Volksverpetzer oder Uebermedien, die ihre oppositionelle Haltung gegenüber rechter Politik zum Ausdruck bringen.

\section{VII.4.3 Homophilie}

Homophilie beschreibt die Bildung von Gruppen innerhalb von Netzwerken («gleich und gleich gesellt sich gern»). Wenig überraschend brachte auch die qualitative Analyse die Erkenntnis, dass gleichgesinnte Medien nicht nur in enger Beziehung zueinanderstehen, sondern sich auch gegenseitig befördern. Dies zeigt sich deutlich anhand der Sub-Kategorie Verlinkung deutungsmächtiger Alternativmedien. Darunter ist zu verstehen, dass besonders resonanzreiche alternative Nachrichtenmedien (im Sinne starker Knoten innerhalb der Netzwerke) von alternativen Nachrichtenmedien geringerer Reichweite wie auch einzelnen Pseudo-Journalist_innen, die eine laienhaften Charakter haben, geteilt werden. $\mathrm{Zu}$ diesen Top-Knoten (im Sinne hoher Eingangsgrade) zählen insbesondere deutsche alternative Nachrichtenmedien wie Tichys Einblick, Russia Today Deutsch, Journalistenwatch, Cicero und Junge Freiheit, die Typ I alternativer Nachrichtenmedien repräsentieren (wie teilweise auch Typ II im Fall von Russia Today), Watchdog-Medien, die dem linken politischen Spektrum und am ehesten Typ IV zuzuordnen sind (Volksverpetzer, BILDblog, Uebermedien), und selten Nachrichtenmedien des Typs III (Kontrast, Junge Welt). Diese Nachrichtenmedien im Sinne starker Knoten werden durch deren Verlinkung über andere Accounts noch weiter in ihrer Aufmerksamkeit befördert und gewinnen somit an Deutungsmacht. Sie sind gleichbedeutend $\mathrm{zu}$ einem hohen Masse jene Medien, die den alternativen Twitter-Diskurs leiten. So erreichen auch laienhaftere Accounts, z. B. ohne eigene Newswebsite, erhöhte Reichweiten und profitieren von der Vernetzung mit den Big Playern. Ein Beispiel hierfür ist der Twitter-Account deutsch365, der durch Retweets ebendieser Knoten Reichweiten (im Sinne von Follower_innen und Retweets) erzielt: «!! !!Geewalttaten: Über 70 Prozent der Verdächtigen sind Ausländer - JUNGE FREIHEIT https://t.co/32zGwNnJM7» ${ }^{181}$, darunter auch länderübergreifende Retweets, wie in diesem Fall des österreichischen Alternativmediums Unzensuriert: «Nach der Wahl ist vor der Wahl: Gute Ausgangslage für FPÖ-Unzensuriert https://t.co/SslyjYTnWo» ${ }^{182}$. Als weiteres Beispiel kann der Account wachjetztauf genannt werden, der ebendieser Logik folgt und etablierte alternative Nachrichtenmedien verlinkt (z. B. Epoch Times, Sputnik, Anonymous etc.). Diese Logik der

181 https://jungefreiheit.de/politik/deutschland/2019/gewalttaten-ueber-70-prozent-der-verdaech tigen-sind-auslaender/ (Junge Freiheit, 18.09.2019)

182 https://www.unzensuriert.at/content/78837-nach-der-wahl-ist-vor-der-wahl-gute-ausgangslage-fuer-fpoe/ (Unzensuriert, 02.10.2019) 
Vernetzung im Sinne einer erhöhten Popularität etablierter Alternativmedien war vorwiegend bei Typ I feststellbar. In ähnlicher Weise zeigte sich im Zuge der Analyse auch eine antizipierte Gemeinschaftsbildung durch einen gemeinsamen «Gegner». Gemeint ist damit eine Vernetzung von Akteur_innen, mit dem Ziel, eine oppositionelle Haltung zu verdeutlichen. Fallweise kann es in diesem Zusammenhang vorkommen, dass sich auch alternative Nachrichtenmedien unterschiedlicher Typen gegenseitig verlinken, wenn in den geteilten Tweets eigene Narrative bedient werden: So verlinkt beispielsweise das verschwörungstheoretische Alternativmedium KenFM einen Beitrag von BILDblog, einem politisch links positionierten Media-Watchdog, der vor allem für Aufklärung einsteht: «RT @BILDblog: Sitzt bei@BILD eigentlich niemand in der Redaktion, der ein bisschen nachdenkt und dann sagt: «Moment mal, Leute, das können wir so nicht bringen. Wir gefährden sonst einen Polizeieinsatz bei einem Terroranschlag!’? https://t.co/Ryow3wSB57 \#Repost \#halloo910 https://t.co/VkQcreiJvD» ${ }^{183}$. Einen gemeinsamen Feind stellt in diesem Fall die deutsche Boulevard-Zeitung BILD dar. Der Tweet bezieht sich auf einen Beitrag der BILD Online, in dem auf die Vorbereitung eines Polizeieinsatzes hingewiesen wird - unter genauer Angabe des Strassennamens. Obwohl die alternativen Nachrichtenmedien KenFM und BILDblog unterschiedliche Ausrichtungen haben, finden sie einen gemeinsamen Nenner durch die Kritik des Mainstream-Boulevard-Journalismus.

Unter der Sub-Kategorie Gleich und gleich gesellt sich gern: Aufruf zur Vernetzung wird eine aktive, mobilisierende Vernetzung subsumiert. Dies erfolgt beispielsweise, indem innerhalb von Tweets eine Vielzahl gleichgesinnter alternativer Nachrichtenmedien bzw. deren Accounts verlinkt werden, wie in diesem Beispiel: «@NachDenkSeiten@IvanRodionov_@TeamKenFM @dieLinkeEP @IMVErlangen @ berlin_vvn_bda@RT_Deutsch@JasminKosubek@ndaktuell@HellePanke@netzpolitik @IALANAgermany@jungewelt@FlassbeckEcon» ${ }^{184}$. Der Tweet stammt vom Account der alternativen Newssite Weltnetz.tv, die Typ II zuzuordnen ist. Nicht verwunderlich ist daher, dass gleichgesinnte Accounts wie TeamKenFM, NachDenkSeiten oder RT_Deutsch verlinkt werden, wie auch Journalisten dieser Nachrichtenseiten (Ivan Rodionov von RT Deutsch) und weitere Akteur_innen, die sich mit dem Thema Medienkritik auseinandersetzen, darunter auch der Account netzpolitik und das alternative Nachrichtenmedium Junge Welt (Typ III). Die Verlinkung von Die Linke und der selbstbeschriebenen «linken Tageszeitung» Neues Deutschland (ndaktuell) zeigt zudem deutlich, dass sich Nachrichtenmedien des Typs II stärker über gemeinsame Themen vernetzen als über politische Grundhaltungen. Die Annahme, dass verschwörungstheoretische Medien grundsätzlich politisch rechtspositioniert sind, kann daher nicht bestätigt werden. Ein ähnliches Bild ergibt sich

183 https://twitter.com/i/web/status/1182245122890764290 (BildBlog, 10.10.2019)

184 https://twitter.com/i/web/status/1182595948884774912 (WeltnetzTV, 11.10.2019) 
auch bei Typ IV alternativer Nachrichtenmedien, wenngleich hier ein stärkerer Bezug zum professionellen Journalismus gegeben ist. Dies ist dadurch zu begründen, dass alternative Nachrichtenmedien des Typs IV zwar die Kommerzialisierung des klassischen Mediensystems ablehnen, aber für qualitativ hochwertigen Journalismus einstehen, der kritisch ist und als Vierte Gewalt agiert, wie an diesem Beitrag der Republik ersichtlich: «RT @m_hof: Dok-Film 〈Die Vierte Gewalt> mit \#EchoderZeit, @derbund, @watson_news, @RepublikMagazin - heute 22:25 Uhr-3Sat https://t.co/CbHrjkJvn1 ${ }^{185}$. Die Republik nimmt in diesem Fall mit Echo der Zeit (SRF) sowohl Bezug auf den öffentlich-rechtlichen Rundfunk wie auch traditionelle Medien (Der Bund) und Online-Medien (Watson) des Mainstreams, indem auf einen gemeinsamen Beitrag über den Journalismus als Vierte Gewalt von unterschiedlichen Schweizer Medien im öffentlich-rechtlichen Rundfunk verwiesen wird.

Die letzte Sub-Kategorie des Themas Homophilie ist die Verteidigung Gleichgesinnter. Diese Kategorie verdeutlicht eine Art Solidarität unter gleichgesinnten Twitter-Akteur_innen: «RT @leykan92: @derStandardat 〈rechtsextrem>In welchem Artikel ruft @Info_DIREKT zu politischer Gewalt auf, heißt diese gut, oder unternimmt Anstrengungen die verfassungsmäßige Ordnung der Republik Österreich abzuschaffen? Bitte um Aufklärung.» ${ }^{186}$ In diesem Tweet einer Privatperson wird die österreichische Nachrichtenseite Info-DIREKT in Schutz genommen, da sie vom österreichischen Leitmedium Der Standard als «rechtsextrem» bezeichnet wurde. Ebenfalls liess die Analyse eine solidarische Haltung alternativer Nachrichtenmedien mit politisch Gleichgesinnten (Parteien oder Politiker_innen) erkennen. Vor allem Typ I, der eine politische Rechtsorientierung aufweist und entsprechend stark mit rechtspopulistischen Parteien und deren Akteur_innen vernetzt ist, offenbart diese Haltung. $\mathrm{Zu}$ beobachten ist dies z. B. anhand eines Tweets der österreichischen alternativen Nachrichtenseite ertaunlich.at, in dem sich (zumindest latent) eine Unterstützung des ehemaligen FPÖ-Politikers HC Strache bzw. seiner Frau Philippa Strache erkennen lässt: «Das scheint wohl in Vergessenheit geraten zu sein! https://t.co/RliztfoWLO Grund für mein in Erinnerung rufen ist, weil sich der ORF aufseiner Internetplattform so ausführlich mit einem angeblichen Gehalt von Philippa Strache beschäftigt. https://t.co/vkVt20lo4l ${ }^{187}$. Eine Verteidigung Philippa Straches findet insofern statt, als auf den Fall der Ex-Geschäftsführerin der Wiener Kinder- und Jugendbetreuung verwiesen wird, die sich vor dem Rechnungshof verteidigen musste - der Fall von Strache wird somit relativiert.

185 https://twitter.com/i/web/status/1183805926362566656 (Republik, 14.10.2019)

186 https://twitter.com/i/web/status/1186409333040517121 (Info Direkt, 21.10.2019)

187 https://www.krone.at/1831098 (Kronen Zeitung, 03.10.2019) 


\section{VII.4.4 Länderübergreifende Vernetzung}

Wie bereits im Zuge der Netzwerkanalysen ersichtlich wurde, konnten fallweise länderübergreifende Vernetzungen zwischen alternativen Nachrichtenmedien identifiziert werden. Dies ist vor allem bei Nachrichtenmedien des Typs I der Fall, die dadurch ihre Resonanz länderübergreifend ausweiten. Herauszustreichen ist in diesem Zusammenhang vor allem die länderübergreifende Vernetzung aufgrund politischer Gleichgesinnung. Insofern handelt es sich bei diesen Relationen häufig um politisch konnotierte Tweets, mit Bezug auf die Politik des rechten Flügels. So verlinkt beispielsweise das österreichische Medium InfoDIREKT einen Tweet vom deutschen Account ein_prozent: «RT @ein_prozent: 6.000 Gegendemonstranten und 1.000-2.000 Teilnehmer bei@OFFICIAL_PEGIDA?@MDR_ SN, ist das euer Ernst? \#Lückenpresse \#Pegida \#ddzo10 https://t.co/TcHizZGAKv» ${ }^{188}$. Gleichzeitig wird in diesem Tweet auf die angeblich falsche Berichterstattung des deutschen Leitmediums MDR verwiesen. Auch umgekehrt verlinken deutsche alternative Nachrichtenmedien auf österreichische Pendants, wie in diesem Fall anhand des Tweets von deutsch 365 mit Verweis auf das österreichische Alternativmedium Unzensuriert festgestellt werden kann: «Rendi-Wagner lässt Katze aus dem Sack: 〈Endlich CO2-Steuer〉 und «FPÖ verhindern〉 - Unzensuriert https://t.co/H4cvmu Coic» ${ }^{189}$. Eine Vernetzung rechtspopulistischer Akteur_innen (im Sinne alternativer Nachrichtenmedien und Politik respektive Politiker_innen) trat insbesondere zwischen Deutschland und Österreich zu Tage, während die Schweiz (wie in den Netzwerken bereits ersichtlich) vordergründig eine eigene Community bildet. Es zeigt sich entsprechend deutlich, dass vor allem Rechtspopulist_innen von den Möglichkeiten digitaler Vernetzung Gebrauch machen und gleichbedeutend $\mathrm{Pu}$ blika aus unterschiedlichen Ländern ansprechen.

\section{VII.4.5 Bezugnahme auf Politik/Politiker_innen}

Wie in der eben diskutierten Kategorie der länderübergreifenden Vernetzung ersichtlich, beziehen sich alternative Nachrichtenmedien häufig auf politische Parteien respektive Politiker_innen - einerseits, um gleichgesinnte Politiker_innen zu unterstützen (vgl. hierzu die Sub-Kategorie Verteidigung Gleichgesinnter), und andererseits, um die Opposition zu diffamieren (vgl. hierzu die Sub-Kategorie Diskreditierung der Opposition). Da beide Subkategorien bereits in einem anderen Kontext angesprochen wurden, soll hier nur mehr kurz darauf eingegangen und dennoch deren Relevanz hervorgehoben werden. Es handelt sich hierbei um ein

188 https://twitter.com/i/web/status/1186240055800549383 (ein_prozent, 21.10.2019)

189 https://www.unzensuriert.at/content/77423-randi-wagner-laesst-katze-aus-sack-endlichco2-steuer-und-fpoe-verhindern-als-programm/ (Unzensuriert, 15.09.2019) 
wesentliches Kommunikationsmittel alternativer Nachrichtenmedien, insbesondere des Typs I. Bei keinem der anderen Typen wurde ein derart starker und vor allem wertender Bezug zu Politik und politischen Akteur_innen festgestellt. Positive Bezugnahmen finden sich insbesondere gegenüber (rechts-)konservativen respektive populistischen Akteur_innen, z. B.: «RT @Junge_Freiheit:Großer Jubel auf der Wahlparty der \#AfD über das Ergebnis der \#ltwth19. https://t.co/Q1Xuf $4 \mathrm{KuNz}{ }^{190}$, oder ein Tweet des alternativen Nachrichtenmediums Oesterreich.press: "\#Österreichs Wahlsieger Kurz sagtillegaler Migration den Kampfan-F.A.Z. - Frankfurter Allgemeine Zeitung https://t.co/JjW2ZeNfy5»"

Ebenso lässt sich ein negativ wertender Bezug von diesem Typ I gegenüber politischen Akteur_innen erkennen, hier am Beispiel eines Tweets von Journalistenwatch: «5 grüne Umweltsünden - und noch viel viel mehr grüner Hirnquark https://t.co/ W4M6Z7C9Ks». Ein ähnliches Muster, im Sinne einer wertenden Bezugnahme auf Politik, findet sich auch bei Typ III, wie beispielsweise in einem Beitrag von Rote Fahne News beobachtbar: «Notwendig ist ein aktiver Widerstand zur Rettung der Umwelt GEGEN die Politik der Bundesregierung https://t.co/nrLNUmTODA / \#AlleFuers Klima \#FridaysForFuture \#Klimastreik \#Fridays4Future» ${ }^{192}$. Im Gegensatz dazu kann bei Typ II kein Muster hinsichtlich politischer Gesinnung festgestellt werden, da bei diesem Typ gemeinsame Themen stärker gemeinschaftsbildend wirken. Typ IV nimmt hingegen auf politische Akteur_innen mit einem sachlichen Diskursstil Bezug, wie ein Tweet der Republik verdeutlicht: «RT@RepublikMagazin: Wahlunterlagen erhalten? Jetzt alle Kandidatinnen Ihres Kantons kennen lernen - inklusive inhaltlicher Positionierung, Interessenbindungen und Wahlkampfbudget. \#WahlCH19» ${ }^{193}$.

\section{VII.4.6 Bezugnahme auf gesellschaftliche Akteur_innen/Organisationen}

Abgesehen von einer teilweise stark identifizierten Politik-Affinität bestimmter alternativer Nachrichtenmedien konnten auch Bezugnahmen auf weitere Akteur_innen festgestellt werden. Darunter Relationen gegenüber gesellschaftlichen Akteur_innen und Organisationen, was anhand der Subkategorien Soziale Bewegungen/Organisationen und Influencer_innen verdeutlicht werden soll. Sehr prominent in beiden Kontexten ist der Klimawandeldiskurs, in dem einerseits (und je nach Typ in unterschiedlicher Weise) auf die Klimabewegung Fridays for Future eingegangen wird, andererseits auf deren Repräsentantin (oder Influencerin) Greta Thunberg. Vor allem Typ III, der sich in erster Linie über den Aufruf zum Protest definiert - im Sinne der neuen sozialen Bewegungen der 1960er- und 1970er-

\footnotetext{
190 https://twitter.com/Junge_Freiheit/status/1188507410606018561 (Junge Freiheit, 27.10.2019)

191 https://t.co/JjW2ZeNfy5 (Oesterreich.Press, 08.10.2019)

192 https://twitter.com/rotefahnenews/status/1174750528284385288 (Rote Fahne News, 19.09.2019)

193 https://twitter.com/i/web/status/1180459960360148992 (Republik, 05.10.2019)
} 
Jahre - berichtet positiv wertend über die Bewegung Fridays for Future und wird in diesem Kontext auch retweetet: «RT @mlpdde: Berichtvon @rotefahnenews-TV zum Protest-und Streiktag der Arbeiter-, Jugend-und Umweltbewegung der am 20.09.19 stattgefunden hat. \#AllefuersKlima \#FridaysForFuture \#GlobalClimateStrike» ${ }^{194}$. Insbesondere die genutzten Hashtags \#AllefuersKlima und \#FridaysForFuture deuten von einer unterstützenden Haltung. Auch beim Media-Watchdog Volksverpetzer (Typ IV) zeigt sich eine positive Grundhaltung gegenüber der Klimabewegung, während gleichzeitig die Berichterstattung des Boulevard-Journalismus kritisiert wird: «RT @Volksverpetzer: Eine \#BILD-Schlagzeilevon 1975 soll angeblich <beweisen, dass die derzeitige \#Rekordhitze in Wahrheit gar kein Rekord sei. Doch auch einer 45 Jahre alten BILD-Schlagzeile sollte man nicht vertrauen. https://t.co/pLIsLnIF w2 \#FridaysForFuture» ${ }^{195}$. Deutlich zu erkennen war im Datenmaterial auch ein Verweis alternativer Nachrichtenmedien des Typs I und zum Teil auch des Typs II auf die Klimabewegung, allerdings eindeutig negativ konnotiert, kritisierend und zum Teil auch diffamierend, wie an diesem Beispiel von Deutschland Kurier deutlich wird: «+++〈Kindersoldaten〉von @georgesoros \& Co. +++ Heute ist wieder «Klimastreik〉 angesagt. Diesmal sogar weltweit! Wer steckt eigentlich hinter den generalstabsmäßig durchorganisierten Klima-Aufmärschen? Der \#DeutschlandKurier deckt auf: \#FridaysForFurture https://t.co/dILuEQmeGo https://t.co/T2CkvM3rxe» ${ }^{196}$. Das Nachrichtenmedium kann als Mischtyp von Typ I und Typ II klassifiziert werden. Abgesehen von dem Ziel, die «Mainstreamlügen» aufzudecken, werden - vor allem in diesem Tweet - auch verschwörungstheoretische Tendenzen sichtbar. So wird auf den Philanthropen George Soros hingewiesen, der häufig Teil elitärer Verschwörungstheorien ist. Innerhalb des Tweets wird implizit von einer von Eliten geplanten Organisation der Klimabewegung ausgegangen («Kindersoldaten»), die Frage «wer steckt eigentlich dahinter?» ist dabei typisch für verschwörungstheoretische Denkmuster, die sich das Lüften von Geheimnissen zum Ziel setzen. Abgesehen von der Klimabewegung wird nur selten auf weitere soziale Bewegungen respektive Organisationen verwiesen, beispielsweise von Typ III auf die Frauenbewegung («Feminist Futures Festival) in Essen: Was der Bewegung in der BRD noch fehlt. Ein Gespräch mit Franza Drechsel. Gitta Düperthal in @jungewelt. https://t. co/mzQAqZKhnl ${ }_{\aleph^{197}}$ ), oder von Typ I - der eigenen politischen Gesinnung entsprechend - auf die stark rechtspositionierte Identitäre Bewegung («RT @Junge_Freiheit: Das Verwaltungsgericht Köln hat dem Bundesamt für Verfassungsschutz untersagt,

194 https://t.co/WoMgmDIXHp (Rote Fahne News, 22.09.2019)

195 https://twitter.com/i/web/status/1154782846210453504 (Volksverpetzer, 21.09.2019)

196 https://twitter.com/i/web/status/1175060198970527746 (Deutschland Kurier, 20.09.2019)

197 https://twitter.com/i/web/status/1173524834137104386 (Junge Welt, 16.09.2019) 
die Identitäre Bewegung als «gesichert rechtsextremistische Bestrebung zu bezeichnen. Das Gericht gab dem Antrag auf Erlaß einer einstweiligen Anordnung statt.» ${ }^{198}$ )

Wie bereits erwähnt, konnten auch Bezugnahmen auf gesellschaftliche Akteur_innen abseits von Politik und Wissenschaft identifiziert werden. Diese können zum Teil als Influencer_innen bezeichnet werden, wie etwa im Fall von Greta Thunberg als die identifikationsstiftende Person hinter der Klimajugendbewegung. Die Verweise auf Thunberg folgen dabei einem ähnlichen Prinzip wie jene auf die Bewegung selbst - je nach Alternativmedientyp positiv unterstützend oder negativ abwertend. Die Logik dahinter ist entsprechend stark polarisierend, eine kontroverse Haltung gegenüber dem Thema und der Person zeigt sich de facto kaum. Ersichtlich wird diese Unterstützung beispielsweise bei diesem Retweet von FreieWeltEU mit Bezug auf Volksverpetzer (beide Typ IV): «RT @Volksverpetzer: Die \#BILD lässt einen Politologen mit leicht widerlegbaren Argumenten Stimmung gegen \#GretaThunberg und @FridayForFuture machen und nennt ihn «Forscher〉, um es seriös erscheinen zu lassen.https://t.co/vnFWXHRk73 \#FridaysForFuture \#Greta \#Klimastreik@ Luisamneubauer» ${ }^{199}$. Negativ wertend äussert sich im Gegensatz dazu Typ I/II: «RT @wach_jetzt_auf: ! Alle reden über Greta ! Ziel: Ablenkung erreicht: !! Überall !! Die Einen stolz wie Bolle auf die Kleine, die Anderen machen sich über die Göre lustig Zur selben Zeit, ! Griechenland: Ein neuer <Flüchtlingstsunami> rollt auf uns zu!!» ${ }^{200}$. Dieser Tweet offenbart eine starke Personalisierung der Klimabewegung mit Bezug auf Greta Thunberg, die mit Begrifflichkeiten wie «Göre» diskreditiert wird. Zudem wird die Prominenz der Klimathematik als Versuch des Mainstreams gedeutet, von der Flüchtlingsthematik abzulenken («Flüchtlingstsunami»). Das Klimathema ist in den untersuchten Tweets derart präsent, dass in diesem Kontext auch auf weitere gesellschaftliche Akteur_innen häufig Bezug genommen wird. So beispielsweise auf den Influencer Rezo, einem YouTuber, der vor allem aufgrund politischer Videos wie «Die Zerstörung der $\mathrm{CDU}^{201}$ » Aufmerksamkeit in der Öffentlichkeit erlangte: «RT @Volksverpetzer: Die YouTuberin \#Mai knüpft an @rezomusik an, tut dabei etwas für das Ansehen von YouTube, die Umwelt - \&jeden Einzelnen von uns. Und hat wichtige Wort für die \#Bundesregierung, die am Freitag ihren Plan fürs \#Klima vorlegen will. https://t.co/XOuoWzYhOK \#Greta \#FridaysForFuture» ${ }^{202}$. Während in diesem Tweet erneut positiv auf die Klimabewegung Bezug genommen wird, werden parallel dazu auch Personen des öffentlichen Lebens dafür eingesetzt, eine Kritik an dem Thema zu äussern. Dies erfolgt z. B. in einem Tweet von Cicero Online (Typ I), in dem auf die kritische Haltung gegenüber Thunberg durch

198 https://twitter.com/i/web/status/1176884778097872896 (Junge Freiheit, 25.09.2019)

199 https://twitter.com/i/web/status/1178607984110444545 (Volksverpetzer, 30.09.2019)

200 https://twitter.com/i/web/status/1176247993261068289 (wach_jetzt_auf, 25.09.2019)

201 https://www.youtube.com/watch?v=4Y1/ZQsyuSQ (Stand: 22.10 .2020 )

202 https://twitter.com/i/web/status/1173876257869750272 (Volksverpetzer, 17.09.2019) 
den deutschen Kabarettisten Dieter Nuhr verwiesen wird: «RT @cicero_online: Ist @dieternuhr, Persona non Greta? Die Frage <Was darf Kabarett?, führt nicht weiter. In der Empörungskultur istes skandalös, Furcht, Panik + Paranoia durch den Bio-Kakao zu ziehen. Aber wir brauchen das, um dem ideologischen Notstand zu entkommen» ${ }^{203}$. Hier wird einerseits implizit der Kabarettist verteidigt und andererseits eine «Empörungskultur» in der Gesellschaft kritisiert.

\section{VII.4.7 Bezugnahme auf Expert_innen/Wissenschaftler_innen}

Abgesehen von Politiker_innen und weiteren Personen des öffentlichen Lebens werden auch starke Bezüge von alternativen Nachrichtenmedien auf Expert_innen und Wissenschaftler_innen sichtbar. Interessant ist dahingehend vor allem, wie auf diese Personen Bezug genommen wird, zumal vor allem Typ I und Typ II auch eine wissenschaftskritische Haltung (mit Bezug auf den Mainstream) einnehmen. Im Zuge der Analyse resultierten zwei Subkategorien: einerseits Legitimation durch wissenschaftliche Expertise, andererseits Expert_innenwahl abhängig vom Narrativ. Die Legitimation durch wissenschaftliche Expertise bezieht sich auf den Verweis auf Expert_innen und Wissenschaftler_innen, um Aussagen alternativer Nachrichtenmedien zu belegen. Expert_innen fungieren dadurch als legitimationsstiftend, wie sie in gleicher Weise zur Legitimation der politischen und medialen Öffentlichkeit - dem Mainstream - auftreten (vgl. hierzu Imhofs (1996) Theorie sozialen Wandels). Dieser Befund konnte über alle Typen alternativer Nachrichtenmedien hinweg beobachtet werden, wonach der Verweis auf Expert_innen als Beleg für die eigene Berichterstattung respektive Grundhaltung dient, wie etwa in folgendem Tweet von Volksverpetzer (Typ IV) ersichtlich: «RT @Volksverpetzer: Die Rechtsextremismus-Expertin @Natascha_Strobl erklärt, wie Faschisten einige Medien in der Causa \#Groenemeyer dazu manipuliert haben, einen demokratischen Konsens in Frage zu stellen. Diese Strategien stecken dahinter. https://t. co/eDLS18IEQu@Gegen_die_AfD@georgrestle»204. Als weiteres Beispiel kann folgender Tweet von Tichys Einblick (Typ I), mit Verweis auf den Historiker Hubertus Knabe, aufgeführt werden: «RT @reitschuster: Der 〈Antifaschismus〉, zu dem sich heute viele, auch Journalisten, lautstark bekennen, und für den sogar im Bundestag geworben wird, ist ein historisch vergifteter, kommunistischer Kampfbegriff,warnt @ hubertus_knabe. Leider scheint das heute weit vergessen https://t.co/tAKsSQXZKr» ${ }^{205 .}$ Auch bei Typ III (Junge Welt) lässt sich dieses Phänomen feststellen: «RT @FuocoSavinelli: Der kubanische Botschafter spricht bei \#UnblockCuba in der Ladengalerie der @jungewelt. Rund 100 Anwesende beschließen Kampagne gegen die verschärfte US-Blo-

203 https://twitter.com/i/web/status/1183348684102213632 (Cicero Online, 13.10.2019)

204 https://twitter.com/i/web/status/1173535342319886341 (Volksverpetzer, 16.09.2019)

205 https://twitter.com/reitschuster/status/1180071877047455744 (Reitschuster, 04.10.2019) 
ckade https://t.co/EabHmQ9aul» ${ }^{206}$. Abgesehen von einer legitimierenden Funktion wurde im Rahmen der Analyse deutlich, dass je nach Narrativ der alternativen Nachrichtenmedien unterschiedliche Expert_innen herangezogen werden, oder eben auch in kritischer, distanzierter Weise auf diese verwiesen wird, so beispielsweise die Sezession: «RT @Recherche_Halle: Es ist noch keine zwei Stunden her und linksaußen <Rechtsextremismusexperte〉 Matthias Quent instrumentalisiert die Tat von \#Halle, um Stimmung gegen@ein_prozent und @IBDeutschland zu machen. Seine Spekulationen passen zu seinem wissenschaftlichen Anspruch... Erbärmlich. https://t. co/9M69JmgQKe» ${ }^{207}$. Die Verwendung von Anführungszeichen mit Verweis auf den Experten verdeutlicht die abwertende Haltung ihm gegenüber. Die Instrumentalisierung von Expert_innen und Wissenschaftler_innen zur Stützung der eigenen Narrative lässt sich in erster Linie dann feststellen, wenn von unterschiedlichen alternativen Nachrichtenmedien auf dieselben wissenschaftlichen Studien verwiesen wird, diese allerdings in unterschiedlicher Weise ausgelegt werden. Beispielhaft sei diesbezüglich ein Verweis auf eine Studie des Reuters Institute Oxford zum öffentlich-rechtlichen Rundfunk erwähnt: Während ein_prozent (Typ I) die mangelnde Unabhängigkeit des öffentlich-rechtlichen Rundfunks bestätigt sieht («Eine Studie des @risj_oxford über den öffentlich-rechtlichen Rundfunk in acht europäischen Ländern bestätigt: Die \#GEZ-Medien \#ARD, \#ZDF, \#Deutschlandfunk sind nicht neutral. \#hoeckeinterview» ${ }^{208}$ ), verweist Uebermedien (Typ IV) auf einen Befund derselben Studie, wonach der öffentlich-rechtliche Rundfunk nicht nur ein politisch links positioniertes Publikum bedienen solle («RT @uebermedien: Beweist eine@risj_oxford-Studie, dass \#ARD und \#ZDF mit ihren Nachrichten nur noch ein linkes Publikum erreichen? Nein: https://t.co/U7ueyb4GP7 @NZZ @FAZ_Feuilleton $\left.{ }^{209}\right)$. Beide Tweets - mit unterschiedlichem Narrativ - nutzen zudem denselben Hashtag \#hoeckeinterview, der auf ein Interview mit AfD-Politiker Björn Höcke verweist, der offenbar in einem Interview auf die Studie Bezug nahm. Die Kategorie Bezugnahme auf Expert_innen/Wissenschaftler_innen verdeutlicht insgesamt, dass Expert_innen zum Zweck der alternativen Nachrichtenmedien instrumentalisiert werden. Dabei findet eine dichotome Unterscheidung zwischen richtigen (das eigene Narrativ unterstützenden) und falschen (das eigene Narrativ kritisierenden) Expert_innen statt. Aus normativer Sicht ist dies vor allem mit Blick auf die Logiken von Social-Media-Plattformen problematisch, wenn über kurze Tweets Aussagen verbreitet werden, die potentiell aus dem Kontext gerissen und durch schnelle Klicks geteilt oder gelikt werden, ohne sie vorher genauer studiert zu haben.

206 https://twitter.com/i/web/status/1174773541436346370 (Savinelli, 19.09.2019)

207 https://twitter.com/i/web/status/1181924697711284226 (Recherche Halle, 09.10.2019)

208 https://twitter.com/i/web/status/1173940726398365696 (ein_prozent, 17.09.2019)

209 https://twitter.com/i/web/status/1174352644984512516 (Uebermedien, 18.09.2019) 


\section{VII.4.8 Bezugnahme Typ I - Mainstream}

Die nachfolgenden Kategorien beziehen sich auf die Relationen zwischen alternativen und professionellen Nachrichtenmedien (dem Mainstream). Diese gegenseitigen Bezugnahmen sind vor allem deswegen interessant, weil sie einen tieferen Einblick in die strukturellen Netzwerkbeziehungen gewähren, die vermuten lassen, dass es Beziehungen zwischen den beiden Lagern gibt. Daher wird folgend jeder Typ alternativer Nachrichtenmedien einzeln beschrieben, da sich die Logiken diesbezüglich stark voneinander unterscheiden. Vor allem bei Typ I («Aufdecker der Mainstreamlügen») konnten vielfältige Verbindungen zur politischen und medialen Öffentlichkeit identifiziert werden, definieren sie sich schliesslich stark durch ihre relationale, oppositionelle Haltung zum Mainstream. Die erste Sub-Kategorie bezieht sich dabei auf eine negative, diffamierende Bezugnahme auf Mainstreammedien. Die Kategorie lässt erkennen, dass Relationen zwischen Typ I und Mainstreammedien vor allem dann auftreten, wenn diese negativ bewertet werden und durch die Bezugnahme selbst eine oppositionelle Haltung deutlich gemacht werden soll. Insofern wird an dieser Stelle bestätigt, dass rein strukturelle Netzwerkverbindungen zwischen diesem Typ und der medialen Öffentlichkeit primär nicht auf einer bestätigenden Haltung basieren. Erkennen lässt sich dies beispielsweise in einem Retweet von Info-DIREKT mit Verweis auf das österreichische Nachrichtenmedium Der Standard: «RT @leykan92: @derStandardat 〈rechtsextrem〉. In welchem Artikel ruft@Info_DIREKT zu politischer Gewalt auf, heißt diese gut, oder unternimmt Anstrengungen die verfassungsmäßige Ordnung der Republik Österreich abzuschaffen? Bitte um Aufklärung.» ${ }^{210}$ Häufig werden professionelle Medien zudem als «Lügenpresse» diskreditiert, indem auf das vermeintliche Verschweigen von Tatsachen hingewiesen wird. So verlinkt beispielsweise die Sezession einen Beitrag von Die Zeit, in dem auf das Problem von Rechtspopulismus verwiesen wird: «RT @lichtmesz: Erzählungen! Nix als Erzählungen! Kein Bezug zur Wirklichkeit! https://t.co/wvj7WAIEAU» ${ }^{211}$.

Interessanterweise zeigte sich auch bei Verlinkungen von URLs professioneller Nachrichtenmedien eine positive Bezugnahme auf Mainstreammedien - vor allem aufgrund einer Unterstützung der eigenen Narrative als weitere Sub-Kategorie. Insbesondere Boulevardmedien werden häufig verlinkt, da diese den Nachrichtenwert der Skandalisierung, vor allem im Migrationskontext, stark bedienen. Als Beispiel sei ein Tweet des deutschen alternativen Nachrichtenmediums Politikversagen (Twitter-Account: staatsversagen) mit Verweis auf die österreichische Kronen Zeitung erwähnt: «Afghane bekam nicht, was er wollte - und stach zu https://t.

210 https://twitter.com/i/web/status/1186409333040517121 (Leyhz92, 22.10.2019)

211 https://t.co/wvj7WAIEAU (Zeit Online, 11.10.2019) 
co/17ZZQx6GMY» ${ }^{212}$. Seltener findet auch ein Verweis auf Qualitätsmedien statt, wenn das eigene Narrativ gestützt wird, so beispielsweise ein Tweet von erstaunlich.at mit Verlinkung auf den österreichischen Kurier: «Man glaubt es kaum - persönliche Haftung für einen Politiker. Ein solches Urteil sollte Schule machen!» ${ }^{213}$

Abgesehen von Mainstreammedien wird von diesem Typ I alternativer Nachrichtenmedien auch Bezug auf den politischen Mainstream genommen. Wie bereits erörtert, findet dabei eine «negative Bezugnahme auf linkspositionierte Politik statt (z. B.: @Gruene_Austria Ich lese bei den Grünen nichts über derartige Beiträge, die in letzter Zeit fast täglich erscheinen. Fast kein Tag ohne eine neue Meldung über die Grünen.» (Tweet von erstaunlich.at mit Verweis auf einen Artikel vom Boulevardmedium Österreich ${ }^{214}$, in dem über die «Grüne Korruptionsaffäre» berichtet wird) und eine positive Bezugnahme auf rechts- oder konservativ-positionierte Politik (z. B. die Junge Freiheit: «Österreichs Ex-Kanzler Sebastian Kurz [ÖVP] hat vor einer neuen Flüchtlingswelle gewarnt. Er kritisierte Spanien und Italien für deren «Umkehr in der Migrationspolitik». Daß beide Länder wieder ihre Häfen für Migranten öffneten, sei das falsche Signal.» ${ }^{215}$ )

\section{VII.4.9 Bezugnahme Typ II - Mainstream}

Obwohl Typ I und Typ II einige bereits erörterte thematische Überschneidungen aufweisen, zeigten sich die Bezüge zum Mainstream bei Typ II nicht so deutlich wie bei Typ I. Dies mag auch daran liegen, dass Typ II in der Twittersphäre nicht in dem Ausmass vertreten ist wie Typ I. Dennoch konnte auch bei diesem Typ bestätigt werden, dass professionelle Nachrichtenmedien vor allem dann positiv wertend verlinkt werden, wenn sie die eigenen Narrative stützen, oder - in gegenteiliger Logik -, um die Opposition deutlich zu machen, im Sinne einer negativen Wertung. Ersteres wird z. B. anhand eines Tweets von Team KenFM ersichtlich, in dem auf einen Artikel von Der Spiege ${ }^{216}$ verwiesen wird: «Hillary Clinton agiert aktuell im Hintergrund des US-Wahlkampfes https://t.co/fyPlUFoyNo Ihre wahre Gedankenwelt sollte nie vergessen werden!» ${ }^{217}$. Das verschwörungstheoretische Narrativ wird insbesondere dadurch deutlich, dass von einer im «Hintergrund» agierenden Rolle Clintons ausgegangen wird, obwohl der Spiegel-Artikel selbst den Titel

212 https://www.krone.at/2024423 (Kronen Zeitung, 17.10.2019)

213 https://t.co/4KdanALPpF (Der Kurier, 02.10.2019)

214 https://t.co/heK61bzlrK (Oe24.at, 21.09.2019)

215 https://twitter.com/i/web/status/1176153257649065986 (Junge Freiheit, 23.09.2019)

216 https://www.spiegel.de/consent-a-?targetUrl=https\%3A\%2F\%2Fwww.spiegel.de\%2Fpolitik $\% 2$ Fausland\%2Fhillary-clinton-warnt-vor-russischer-unterstuetzung-fuer-kandidatin-tulsigabbard-a-1292321.html\&ref=https\%3A\%2F\%2Fwww.google.com\%2F (Stand: 23.11.2020)

217 https://t.co/FmjnVCCxsH (Team KenFM, 21.10.2019) 
«Hillary Clinton warnt vor russischer Unterstützung für Kandidatin bei US-Wahl» trägt. Im Artikel selbst wird auf den Diskurs zwischen Clinton und Tulsi Gabbard eingegangen, wonach Gabbard Clinton via Twitter als «Kriegstreiberin» bezeichnete. Wie auch bei Typ I werden zudem skandalisierende Beiträge von Boulevardmedien dazu genutzt, um eigene Haltungen zu unterstreichen, so z. B. WakeNewsRadio mit Verweis auf tag24. de: «Flüchtlinge: Schwere Randale bei Registrierung in der EU, Unterkünfte angezündet» ${ }^{218}$. Weiter werden URLs von professionellen Nachrichtenmedien (wie auch bei Typ I) dazu verwendet, um die oppositionelle Haltung zum «Mainstream» herauszustreichen, wie etwa in folgendem Retweet von KenFM deutlich wird:

RT @norberthaering: Der arme Albrecht Müller! Kein Mensch interessiert sich laut Süddeutscher Zeitung für ihn. Also muss ihn die SZ auf Seite 1 ausführlich anonym beschimpfen, was nur seine Wichtigkeit beweist. Der Streiflicht-Schreiber sollte sich für seine feige Attacke in Grund und Boden schämen. https://t.co/ e2q8Kobehl $\left.\right|^{219}$

Die Süddeutsche Zeitung wird in diesem Fall abgelehnt, da sie den Mitherausgeber der NachDenkSeiten (ebenfalls Typ II) aufgrund verschwörungstheoretischer Aussagen (u. a. bezüglich 9/11) kritisierte. Es handelt sich demnach gleichzeitig um eine Unterstützung gleichgesinnter Nachrichtenmedien, und eine Differenz zwischen Ingroup und Outgroup (im Sinne von: «Wir gegen die anderen») wird deutlich.

\section{VII.4.10 Bezugnahme Typ III - Mainstream}

Typ III («Aufstand der Zivilgesellschaft») ist - wie bereits erwähnt - wenig präsent auf Twitter. Dennoch wird über einzelne Twitter-Kanäle dieses Typs eine kapitalismuskritische, mobilisierende Linie ersichtlich. Ein Verweis auf Mainstreammedien findet demnach in den seltensten Fällen statt, vielmehr wird auf die politische Öffentlichkeit Bezug genommen, mit einer positiven Grundhaltung gegenüber sozialdemokratischen Ansichten und einer negativen Einstellung gegenüber konservativer respektive rechtspositionierter Politik. So verlinkt beispielsweise das österreichische Nachrichtenmedium Kontrast SPÖ-Politikerin Rendi-Wagner mit gleichzeitiger Kritik an ÖVP und FPÖ: «Weil @rendiwagner gerade die sinnlosen Ausgaben bei der Zusammenlegung der Sozialversicherungen erwähnt hat. Auch in anderen Bereichen prassten \#ÖVP und \#FPÖ mit Steuergeldern. https://t.co/IzmQdQJc2B» ${ }^{220}$,

218 https://t.co/Xf]K3NC94W (tag24, 28.10.2019)

219 https://twitter.com/i/web/status/1173691466092306432 (Team KenFM, 17.09.2019)

220 https://twitter.com/i/web/status/1174415081268027392 (Kontrast, 18.09.2019) 
oder (ebenfalls Kontrast): «Im ORF meint @sebastiankurz gerade, es gäbe nichts mehr zum Aufklären beim \#BVT. Hier zur Auffrischung nur einer von vielen Skandalen um den BVT. \#wahl19» ${ }^{221}$. In diesem Fall wird zwar auf den öffentlich-rechtlichen Rundfunk als Mainstream-Medium Bezug genommen, durch die Verlinkung auf Sebastian Kurz' Twitter-Account erzielt die politische Bezugnahme allerdings grösseres Gewicht, bzw. wird ein Diskurs mit dem ÖVP-Politiker antizipiert. Eine Verlinkung von Mainstreammedien, um Narrative zu bestätigen oder Kritik an professionellen Medien zu äussern, findet bei Typ III nicht in dieser Form statt.

\section{VII.4.11 Bezugnahme Typ IV - Mainstream}

Typ IV («Die seriöse Alternative») bezieht sich - im Vergleich zu den anderen drei Typen - in sehr unterschiedlicher Weise auf den Mainstream. Im Vordergrund stehen dabei Relationen zu professionellen Nachrichtenmedien. Insgesamt konnten zwei Subkategorien identifiziert werden, darunter die Investigative Aufdeckung (kontroverse Haltung) und die Unterstützende Bezugnahme, sofern die Funktion als Vierte Gewalt erfüllt wird. Entsprechend dem Selbstbild von Typ IV nimmt investigativer Journalismus eine wesentliche Rolle ein, auch im Sinne einer Kontrollfunktion der Mainstreammedien. So richtet sich beispielsweise das österreichische Magazin Addendum an das Boulevard-Magazin Österreich, um auf einen Fehler in der Berichterstattung hinzuweisen: «Liebe Kollegen von @Oe24at,wenn wir das richtig interpretieren, habt ihr beim Kopieren unserer Story vergessen, uns auch im letzten Absatz als Quelle zu löschen. \#servicetweet https://t.co/90RwbVlZgz»222. Addendum kritisiert in diesem Fall zwar die mangelnde Quelleneinordnung des Nachrichtenmediums, dies erfolgt allerdings in einem sachlicheren Diskursstil (vor allem im Vergleich zu Typ I). Generell macht Typ IV weniger eine oppositionelle Haltung zum Mainstream stark, wenngleich beispielsweise die Kommerzialisierung grosser Medienhäuser per Selbstverständnis abgelehnt wird. Bezug auf professionelle Nachrichtenmedien wird zudem genommen, wenn eine Kritik des Mediensystems auch vom Mainstream geäussert wird. Beispielhaft sei diesbezüglich ein Tweet der Republik mit Verweis auf das Magazin Schweizer Journalist genannt: «RT @janoschtroehler: Printmedien in der Schweiz: Die statistik des Grauens>,analysiertvon@CR_Sieber.Ein Abwärtstrend, der sich-trotz@Wochenzeitung-Phänomen - nicht mehr umkehren lässt. https://t.co/IR7r4UgbtA» ${ }^{223}$.

Eine Abgrenzung im Sinne einer gegenöffentlichen Position erfolgt daher vergleichsweise seltener. Insofern können auch lobende Worte gegenüber professionellen Nachrichtenmedien ausgesprochen werden, wie an diesem Tweet von

221 https://twitter.com/i/web/status/1174400093891518465 (Kontrast, 18.09.2019)

222 https://twitter.com/i/web/status/1176134203496439808 (Addendum, 23.09.2019)

223 https://twitter.com/i/web/status/1173914738541105152 (Tröhler, 17.09.2019) 
Das Lamm ersichtlich: «Nachtrag zur Läderach-Geschichte: So geht das, @Tagesanzeiger. Danke fürs Erwähnen, @KoponenLinda und @NZZ!»224. Ein gemeinsamer Nenner zwischen Typ IV und professionellen Nachrichtenmedien findet sich folglich hauptsächlich dann, wenn die gemeinsame Funktion als Vierte Gewalt erfüllt wird, wie sich hier deutlich zeigt: «RT@m_hof: Dok-Film 〈Die Vierte Gewalt>mit \#EchoderZeit, @derbund, @watson_news, @RepublikMagazin - heute 22:25Uhr-3Sat https://t.co/CbHrjkJvn1» ${ }^{225}$.

\section{VII.4.12 Media-Watchdogs}

Abgesehen von den vier Typen alternativer Nachrichtenmedien wurde im Datenmaterial ein weiterer Medientyp identifiziert, der bereits als Media-Watchdog eingeführt wurde. Gemeint sind hiermit Nachrichtenmedien, die am ehesten Typ IV zugeordnet werden können, allerdings per Selbstverständnis eine weitere Rolle einnehmen. Ihr primäres Ziel besteht nicht darin, selbst Nachrichten aufzubereiten und zu verbreiten, sondern vielmehr darin, eine Watchdog-Funktion gegenüber dem gesamten Mediensystem einzunehmen. Zentral ist dabei die selbst auferlegte Korrektivfunktion. An dieser Stelle soll spezifisch auf diese Media-Watchdogs eingegangen werden, da sie einen besonderen Stellenwert in Relation zum Mainstream einnehmen. Prägend für die Watchdogs ist eine Überwachung des gesamten Mediensystems, einhergehend mit einer Kritik, dass beispielsweise Tatsachen verdreht würden,wie an diesem Beispiel ersichtlich: «RT@ BILDblog: Greta Thunberg schreibt, man solle Erwachsenen, die Kinder und Jugendliche verspotten und bedrohen, keine Beachtung schenken. Die @welt-Redaktion macht daraus: «Wer ihr Vorgehen zum Klimaschutz nicht billigt, sei keiner Beachtung wert, schrieb sie >: https://t.co/i2ey6ems9D» ${ }^{226}$. Die Überwachung des Mediensystems bezieht sich dabei nicht nur auf professionelle Nachrichtenmedien, sondern auch auf alternative Nachrichtenmedien; so nimmt etwa der BILDblog Bezug auf das alternative Nachrichtenmedium Cicero: «RT @BILDblog: Die@cicero_online-Redaktion hat nun aufunsere Kritik reagiert, die Passage geändert und am Ende des Artikels einen Hinweis veröffentlicht: https://t.co/3PZqwbVgcN \#Nachtrag https://t.co/kTjTB59P4a» ${ }^{227}$.

Die als Media-Watchdogs identifizierten Twitter-Accounts lassen auch eine klare politische Haltung erkennen, die sich - wie bereits erörtert - gegen rechtsorientierte Politik richtet. Es kann in diesem Fall von einem gruppenbildenden Prozess gesprochen werden, wonach ein Bündnis gegen Rechts - als weitere Sub-Kategorie - verfolgt wird: «RT @Volksverpetzer: In diesem Video werden 34 der schlimms-

\footnotetext{
224 https://t.co/GCeLpsgzCZ (NZZ, 14.09.2019)

225 https://twitter.com/i/web/status/1183805926362566656 (Hofmann, 14.10.2019)

226 https://t.co/4gHO23W7ll (BildBlog, 01.10.2019)

227 https://twitter.com/i/web/status/1176530982377263105 (BildBlog, 24.09.2019)
} 
ten \#AfD-Zitate von einfachen Menschen vorgelesen. Dieses Video von @RedereiFM entlarvt den Hass der AfD. Erschreckend! https://t.co/SZ4baCVll1@Gegen_die_AfD@hoge satzbau \#Höcke \#Thueringen» ${ }^{228}$. Dieses Narrativ zeigte sich insbesondere bei Medien wie Volksverpetzer, BILDblog und Uebermedien.

Weiter verfolgen die Media-Watchdogs das Ziel der Aufklärung, indem auf Missstände in der Berichterstattung von Nachrichtenmedien hingewiesen wird - so beispielsweise der BILDblog: «Wie@BILD aus einem Mann mit einem Messer vor einer Synagoge eine Verschwörungstheorie gegen die Berliner Regierung bastelt: https://t. co/bHPhIUezx4» ${ }^{229}$. Die Media-Watchdogs nehmen insofern eine wesentliche journalistische Funktion im Sinne einer Vierten Gewalt ein. In Abgrenzung zu den vier Idealtypen alternativer Nachrichtenmedien, die vor allem selbst Nachrichten aufbereiten und verbreiten, sehen sie sich stärker in der Funktion einer Medien-Metakritik. Inhaltlich können sie am ehesten Typ IV zugeordnet werden.

\section{VII.4.13 Bezugnahme Mainstream - Alternativmedien}

Die letzte Kategorie widmet sich den Relationen zwischen Mainstreammedien im Sinne professioneller Informationsmedien und alternativen Nachrichtenmedien. Im Zuge der Analyse stellte sich heraus, dass aktive Bezugnahmen von professionellen Nachrichtenmedien auf alternative Nachrichtenmedien äusserst selten vorkommen. Dies kann mehrere Gründe haben: Zum einen bildet der Mainstream im Sinne der «öffentlichen Meinung» eine eigene Community, die primär untereinander referenziert. Zum anderen scheint es eine bewusste Haltung professioneller Nachrichtenmedien zu sein, Alternativmedien keine Bühne bieten zu wollen - vor allem dann nicht, wenn desinformative Beiträge von bestimmten Seiten geteilt wurden. In diesem Kontext würde die Thematisierung von «Fake News» im Sinne einer gesellschaftlichen Aufklärung zwar durchgeführt, allerdings ohne Referenzen auf einschlägige alternative Nachrichtenmedien. Während URLs alternativer Nachrichtenmedien de facto nicht in den Tweets professioneller Nachrichtenmedien verlinkt werden, findet fallweise ein Bezug auf diese in Form von Mentions (@) statt. Dies erfolgt allerdings vorwiegend in Bezug auf alternative Nachrichtenmedien des Typs IV respektive Media-Watchdogs, die schliesslich selbst keine dezidiert ablehnende Haltung gegenüber professionellen Nachrichtenmedien einnehmen. So berichtigt beispielsweise die Schweizer NZZ einen eigenen Beitrag, nachdem Uebermedien auf einen Fehler in der Berichterstattung hingewiesen hatte: «@uebermedien@risj_oxford@FAZ_Feuilleton Wir haben den Text überarbeitet. Die erste Version war so nicht richtig. Wir entschuldigen uns dafür.

228 https://twitter.com/i/web/status/1187302934901657601 (Volksverpetzer, 24.10.2019)

229 https://t.co/epWekDUCSc (BildBlog, 09.10.2019) 
https://t.co/CZXZKuhnaS» ${ }^{230}$. Als weiteres Beispiel kann ein Tweet der deutschen Wochenzeitung Der Freitag herangezogen werden, in dem auf das deutsche alternative Nachrichtenmedium Krautreporter (Typ IV) verwiesen wird: «\#ExtinctionRebellion fordert mit ihrem friedlichen Ungehorsam etablierte Klimaschützer (und das ganze Land) heraus. @gri_mm über die \#Klimabewegung, die Grüne provoziert. Via @krautreporter \#Netzlese https://t.co/YpzQ7qGQIf ${ }^{231}$. Gesamthaft betrachtet sind Verweise wie diese allerdings eine seltene Ausnahme - vor allem im Vergleich zu umgekehrten Bezugnahmen, wie in den vorigen Sub-Kapiteln beschrieben. Abgesehen davon gibt es im Raum Schweiz eine Ausnahme, wobei auf ein alternatives Nachrichtenmedium des Typs I, nämlich die Weltwoche, verwiesen wird. Es handelt sich um einen Tweet von Persoenlich.com: «Eines kann ich jetzt schon versprechen: Wenn ich in den Ständerat gewählt werde, setze ich dieses Amt nicht ein, um irgendwelche VR-Mandate zu holen und mich als Lobbyist einkaufen zu lassen), so @ KoeppelRoger, Verleger und Chefredaktorder@Weltwoche. https://t.co/KDdnYi6Txj»"232. Diesbezüglich ist gleichwohl von einem Sonderfall zu sprechen, und zwar dahingehend, dass die Weltwoche zwar per Selbstverständnis eine oppositionelle Haltung gegenüber dem Mainstream einnimmt, allerdings in gewisser Weise Teil des Schweizer Medien-Mainstreams ist, da es sich um ein etabliertes, rechts positioniertes Nachrichtenmedium der Deutsch-Schweiz handelt.

\section{VII.4.14 Zwischenfazit: Relationen alternativer Nachrichtenmedien und theoretische Einordnung}

Bezugnehmend auf die Frage, wie Öffentlichkeit in modernen, digitalen Gesellschaften definiert werden kann, bestätigten die empirischen Analysen die im Theorieteil bereits ausformulierten Thesen bzw. Ansätze: Sowohl Öffentlichkeit - im Rahmen dieser Studie repräsentiert durch professionelle Informationsmedien im deutschsprachigen Raum - als auch Gegenöffentlichkeit - im Sinne von alternativen Nachrichtenmedien ${ }^{233}$ - können nicht als getrennt voneinander zu betrachtende Konstrukte oder gar Räume behandelt werden. Bereits die strukturellen Netzwerkanalysen in Kapitel VII.3 zeigten, dass diese - unabhängig davon,

\footnotetext{
230 https://twitter.com/i/web/status/1175360570905812992 (NZZ, 21.09.2019)

231 https://twitter.com/i/web/status/1181842991218069504 (Der Freitag, 09.10.2019)

232 https://twitter.com/i/web/status/1176375722732138496 (Persoenlich.com, 24.09.2019)

233 Öffentlichkeit und Gegenöffentlichkeit inkludieren selbsterklärend nicht nur journalistische Medien, sondern können beispielsweise auch durch Politikund Expert_innenkulturenabgebildet werden, wie auch - im gegenöffentlichen Bereich -z. B. durch Protestgruppen, Organisationen etc. Im Rahmen dieser Studie wurde aber bewusst auf Nachrichtenmedien als zu untersuchendes Phänomen fokussiert, die gleichbedeutend als empirischer Wirklichkeitsausschnitt gewählt wurden.
} 
ob Follower- oder Retweetstrukturen betrachtet werden - über Beziehungen bzw. Relationen miteinander verbunden sind.

Die qualitative Inhaltsanalyse der Twitter-Posts verdeutlichte, dass «klassische Nachrichtenwerte» wie Polarisierung, Skandalisierung, Personalisierung und Emotionalisierung starke Treiber für Engagement in Form von Favorites (Likes) und Retweets sind. Dabei liess sich im Kontext alternativer Nachrichtenmedien insbesondere beobachten, dass polarisierende Haltungen, wie sie die Definition von Alternativmedien nahelegt, nämlich eine Opposition zum Mainstream darzustellen, Resonanz in der Twittersphäre erzielen. Zudem führen Plattformlogiken zu einer stärkeren Popularität bzw. Deutungsmacht von Akteur_innen, beispielsweise über Mentions oder Retweets, während Hashtags vor allem einen Themenbezug deutlich machen. Wesentlich ist zudem die Logik der Vernetzung, wonach vor allem gleichgesinnte Akteur_innen an Deutungsmacht gewinnen, indem sie sich gegenseitig nicht nur abonnieren, um deren Beiträge zu rezipieren, sondern sich auch gegenseitig verlinken respektive retweeten. Bezugnehmend auf Bourdieus (2013 [1979]) Feldtheorie kann in diesem Zusammenhang von einer Anreicherung an Kapital - nämlich vor allem symbolischem Kapital im Sinne von Deutungsmacht - gesprochen werden. Dabei spielt vor allem im Kontext digitaler Plattformen das soziale Kapital eine wesentliche Rolle, im Sinne eines Gewinnes an Popularität durch soziale Kontakte auf der Plattform. Die einzelnen Knoten innerhalb der Netzwerke demonstrieren gleichbedeutend ihre Positionierung im sozialen Feld nach Bourdieu (2013 [1979]). Starke Knoten, in Form von hohen Eingangsgraden, können als besonders mächtige Akteur_innen der Netzwerke betrachtet werden. Es ist nicht verwunderlich, dass vor allem Politiker_innen und Nachrichtenmedien, die die öffentliche Meinung vertreten - zentrale Knoten innerhalb der Netzwerke darstellen. Ein Blick auf die Communities alternativer Nachrichtenmedien verdeutlicht gleichzeitig, dass auch alternative, gegenöffentliche Akteur_innen gesamthaft betrachtet an Deutungsmacht gewinnen können. Dahingehend stechen vor allem Nachrichtenmedien des Typs I heraus, die sich das Ziel setzen, vermeintliche Lügen professioneller Nachrichtenmedien aufzudecken. Dass sie politisch vorwiegend rechts positioniert sind, zeigt sich in ihren positiven Bezugnahmen gegenüber rechtspopulistischen Politiker_innen, aber auch inhaltlich, indem vor allem rechte Narrative unterstützt werden. Diese inhaltlichen Bezugnahmen verdeutlichen den Habitus innerhalb der Communities, der massgeblich beeinflusst, wie die Akteur_innen rhetorisch argumentieren, um die Erwartungen ihrer Community zu erfüllen - ein wesentliches Element bei Simmels (1968 [1908]) Ausführungen zu den sozialen Kreisen.

Fundamental für die Vernetzung von Akteur_innen ist das Konzept der Homophilie, wonach sich vor allem gleichgesinnte Akteur_innen untereinander vernetzen wie auch gegenseitig verteidigen und weiter oppositionelle Akteur_innen implizit oder explizit abwerten. Insofern wird eine Polarisierung zwischen 
Ingroup und Outgroup deutlich. In diesem Zusammenhang kann auf Simmels (1968 [1908]) soziale Kreise oder Elias' (2006 [1970]) Figurationen verwiesen werden. Simmel (1968 [1908], S. 325f.) beschreibt in diesem Kontext einen «Kollektivismus» innerhalb sozialer Kreise (oder Communities) im Sinne gemeinschaftsbildender Prozesse innerhalb der Gruppe. In Webers (1985 [1922]) Argumentation könnte auch von einem Vergemeinschaftungsprozess durch affektuelles (vorwiegend beruhend auf Emotionen) Handeln gesprochen werden, das eine subjektiv gefühlte Zusammengehörigkeit unter Akteur_innen forciert. Ebendiese emotionalen Bindungen (die gleichzeitig die Logik der Emotionalisierung als Medienrespektive Plattformlogik widerspiegeln und zu Resonanz auf den Plattformen führen) spielen auch bei Elias' (2006 [1970]) Figurationstheorie eine wesentliche Rolle und repräsentieren gleichzeitig das Bedürfnis nach Angehörigkeit in der Gesellschaft. Das «Ich-und-Wir»-Bewusstsein (Elias, 2006 [1970], S. 182) manifestiert sich hauptsächlich durch die Abgrenzung zu den anderen, dem Mainstream, und das Spiel zwischen Macht und Gegenmacht. Die Ausführungen von Elias und Scotson (2002 [1965]) zum Verhältnis zwischen Etablierten und Aussenseiter_innen können auch auf die Relationen zwischen Öffentlichkeit und Gegenöffentlichkeit, also dem politischen und medialen Mainstream und alternativen Nachrichtenmedien, adaptiert werden. Insofern zeigt sich - zumindest bei den ersten beiden Typen alternativer Nachrichtenmedien - vor allem aus Sicht der Alternativmedien eine gefühlte Wahrnehmung als Aussenseiter_in, bzw. zelebrieren sie nahezu ihre oppositionelle Haltung gegenüber unterschiedlichen Themen, die die öffentliche Meinung repräsentieren. Dies erfolgt vor allem durch Beiträge, in denen der Mainstream diskreditiert wird. Sie selbst sehen sich - wie im Rahmen der typologischen Einordnung klar wurde (vgl. Kapitel VII.2) - als eindeutiger Gegenpol zur hegemonialen Öffentlichkeit.

Relationen unter gleichgesinnten Akteur_innen bestehen nicht nur in Bezug auf Nachrichtenmedien des gleichen «Typs». Vielmehr nehmen alternative Nachrichtenmedien auch Bezug auf Politiker_innen und weitere gesellschaftliche Akteur_innen (wie z. B. auch Influencer_innen), Organisationen und Expert_innen aus Wissenschaft und Forschung. Dabei spielt auch eine länderübergreifende Vernetzung eine wesentliche Rolle vor allem für Typ I alternativer Nachrichtenmedien, da bei diesem Typ insbesondere die politische Gleichgesinnung ein wesentliches Element für die Vernetzung darstellt und eine Art Allianz gegen den Mainstream gebildet wird. Dies bestätigt Castells' (2010 [1996]) Annahmen der Netzwerkgesellschaft, die von zunehmender Globalisierung geprägt ist. Die Verlinkung dieser Akteur_innen folgt einer binären Logik, wonach klar zwischen $\mathrm{Zu}$ stimmung und Ablehnung differenziert wird und kontroverse Ansichten nur selten Einzug halten. Insofern werden beispielsweise auch Expert_innen klar dem eigenen Narrativ folgend ausgewählt, um eigene Ansichten zu stützen. 
Die qualitative Analyse veranschaulichte zudem inhaltliche Relationen, im Sinne von Narrativen zwischen Öffentlichkeit und Gegenöffentlichkeit. Dabei wurde sichtbar, dass insbesondere alternative Nachrichtenmedien aktiv auf den professionellen Informationsjournalismus Bezug nehmen, beispielsweise in Form von Retweets (also z. B. der Verlinkung von Beiträgen professioneller Informationsmedien), Mentions (der Erwähnung professionell journalistischer Twitter-Accounts) oder Hashtags mit Bezug zum Mainstream-Journalismus. Ebenso verhält es sich im Übrigen auch mit politischen Akteur_innen und Parteien des Mainstreams, die von alternativen Nachrichtenmedien innerhalb von Tweets erwähnt werden. Diese Relationen zur Öffentlichkeit liegen jedoch in unterschiedlicher Form vor und variieren je nach Typ alternativer Nachrichtenmedien. Der präsenteste Typ I («Aufdecker der Mainstreamlügen») verweist vor allem in negativer bzw. ablehnender Weise auf mediale und politische Eliten, den Mainstream. Dies spiegelt die bereits erwähnte In- bzw. Outgroup-Dynamik wider: Einerseits wird der eigene Gruppen- oder Community-Zusammenhang dadurch gestärkt, dass die innerhalb der Gruppe vertretene Meinung im Sinne eines gruppenbildenden Prozesses weiter betont wird. Gleichzeitig wird die oppositionelle Haltung zur öffentlichen Meinung des Mainstreams verdeutlicht, wodurch ein Abgrenzungsprozess stattfindet. Obwohl in diesem Fall Relationen zwischen Öffentlichkeit und Gegenöffentlichkeit existieren, und diese aus struktureller Sicht nahelegen, dass ein wechselseitiger Diskurs stattfindet (im Gegensatz zur These einer Echokammernbildung), zeigt die interpretative Analyse, dass eine Gruppenpolarisierung durch die gegenseitigen Bezugnahmen noch weiter verstärkt wird. Bezugnahmen von professionellen Nachrichtenmedien auf alternative Nachrichtenmedien finden im Unterschied dazu kaum statt. Dies trifft nur in seltenen Fällen $\mathrm{zu}$, insbesondere aber in Bezug auf alternative Nachrichtenmedien des Typs IV («Die seriöse Alternative»), die selbst demokratische Ziele verfolgen und ihre Opposition gegenüber dem Mainstream primär basierend auf einer Kritik des kommerzialisierten Mediensystems deutlich machen, selbst aber eine positive Grundhaltung gegenüber professionellem, gut recherchiertem Journalismus einnehmen. Abgesehen von den vier identifizierten Typen alternativer Nachrichtenmedien brachte die interpretative Analyse der Tweets zudem das Ergebnis hervor, dass sich Korrektiv-Seiten medienübergreifend das Ziel setzen, das Mediensystem $\mathrm{zu}$ kontrollieren und $\mathrm{zu}$ kritisieren, im Sinne einer Media-Watchdog-Funktion. Diese sind am ehesten Typ IV alternativer Nachrichtenmedien zuzuordnen, wenn auch weniger das Ziel verfolgt wird, selbständig Nachrichten aufzubereiten als vielmehr eine Medien-Metakritik vorzunehmen.

Gesamthaft verdeutlichte die qualitative Analyse der Netzwerkstrukturen die Wichtigkeit interpretativer Verfahren. Eine strukturelle Veranschaulichung von Relationen kann nur dann umfassend eingeordnet werden, wenn Narrative - das Dazwischenliegende - interpretiert werden. Insofern kann festgehalten werden, 
dass Echokammern im Kontext alternativer Nachrichtenmedien zwar definitorisch nicht bestehen, da alternative Nachrichtenmedien selbst auf den Mainstream in ihren Beiträgen verweisen, dies aber vielmehr dazu führen kann, dass eine Polarisierung noch weiter verstärkt wird, wenn die öffentliche Meinung diskreditiert oder ironisiert wird. Pörksen (2018) spricht in diesem Zusammenhang von «Filter-Clashes», wonach polarisierende Grundhaltungen und die Publikation dieser das Problem einer gesellschaftlichen Spaltung noch weiter verstärken können. Dies zeigte sich fallweise im Datenmaterial, vor allem bezugnehmend auf Typ I und Typ II, die schliesslich «Fake News» des Mainstreams respektive Elitenverschwörungen aufzudecken versuchen. In Abgrenzung dazu präsentieren sich Typ III und Typ IV in Relation zur medialen und politischen Öffentlichkeit sachlicher und konstruktiver, indem sie einerseits Missstände aufzeigen, um soziale Gerechtigkeit zu forcieren (Typ III), oder die demokratische Rolle von Nachrichtenmedien verdeutlichen (Typ IV). Eine Untergliederung in stark voneinander getrennte «Echokammern» konnte im analysierten Datenmaterial nicht identifiziert werden, allerdings ist davon auszugehen, dass gerade jene «Filter-Clashes» Polarisierungstendenzen zwischen öffentlichen und gegenöffentlichen Gruppierungen weiter verstärken. 
